\title{
Targeting CD44 receptor-positive lung tumors using polysaccharide-based nanocarriers: influence of nanoparticle size and administration route
}

Victor Jeannot, PharmD ${ }^{\mathrm{a}, \mathrm{b}}$ *, Silvia Mazzaferro, Pharm.D., Ph.D. ${ }^{\mathrm{c}, \mathrm{d}}$, Jonathan Lavaud ${ }^{\mathrm{a}, \mathrm{b}, \mathrm{e}}$, Laetitia Vanwonterghem $^{\mathrm{a}, \mathrm{b}}$, Maxime Henry ${ }^{\mathrm{a}, \mathrm{b}, \mathrm{e}}$, Mélanie Arboléas $^{\mathrm{a}, \mathrm{b}}$, Julien Vollaire $^{\mathrm{a}, \mathrm{b}, \mathrm{e}}$, Véronique Josserand, Ph.D., ${ }^{\mathrm{a}, \mathrm{b}, \mathrm{e}}$, Jean-Luc Coll, Ph.D., ${ }^{\mathrm{a}, \mathrm{b}}$, Sébastien Lecommandoux, Ph.D., ${ }^{\mathrm{c}, \mathrm{d}}$, Christophe Schatz, Ph.D. ${ }^{\text {c,d } \#, ~ A m a n d i n e ~ H u r b i n, ~ P h . D . ~}{ }^{\text {a,b \# }}$

${ }^{a}$ INSERM U823, Institut Albert Bonniot, F-38000 Grenoble, France

${ }^{\mathrm{b}}$ Univ. Grenoble Alpes, Institut Albert Bonniot, F-38000 Grenoble, France

${ }^{c}$ CNRS UMR5629, Laboratoire de Chimie des Polymères Organiques, F-33607 Pessac, France

${ }^{\mathrm{d}}$ Univ. Bordeaux, Bordeaux-INP, F-33607 Grenoble, France

${ }^{\mathrm{e}}$ OPTIMAL small animal imaging facility, F-38000 Grenoble, France

* these authors contributed equally to this work

\# co-last authors

\section{Co-corresponding authors:}

Amandine Hurbin. INSERM/UJF U823, Institut Albert Bonniot, BP170, 38042 Grenoble cedex 9, France. Tel: 334765495 53. Fax: 334765494 13. Email: amandine.hurbin@inserm.fr

Christophe Schatz. CNRS UMR5629, Laboratoire de Chimie des Polymères Organiques, 16 avenue Pey Berland, 33607 Pessac, France. Tel: 335400036 96. Fax: 335400084 87. Email: schatz@enscbp.fr

Fundings: This study was funded by the National Research Agency (ANR project Nanoluc ANR11-BSV5-0018), France Life Imaging (FLI, French program "Investissement d'Avenir"; grant "Infrastructure d'avenir en Biologie Santé", ANR-11-INBS-0006), and Region Rhône-Alpes.

Conflicts of interest: There are no known conflicts of interest associated with this publication.

Word count for abstract: 150

Manuscript word count (body text and figure legends): 4998

Number of references: 33

Number of figures: 6

Number of Supplementary online-only files: 5

\begin{abstract}
Abbreviations: AFM, atomic force microscopy; DLS, dynamic light scattering; DP, degree of polymerization; EPR, enhanced permeability and retention; Hya, Hyaluronan; MFI, mean fluorescence intensity; NP, nanoparticles; Np30, hyaluronan-based nanoparticles of $30 \mathrm{~nm}$ diameter; Np300, hyaluronan-based nanoparticles of $300 \mathrm{~nm}$ diameter; NSCLC, non-small cell lung cancer; PBLG, poly( $\gamma$-benzyl-L-glutamate); RES, reticuloendothelial system; ROI, region of interest; ROP, ring-opening polymerization; SANS, small-angle neutron scattering; TEM, transmission electron microscopy.
\end{abstract}


Specific tumor cell targeting

Hyaluronan

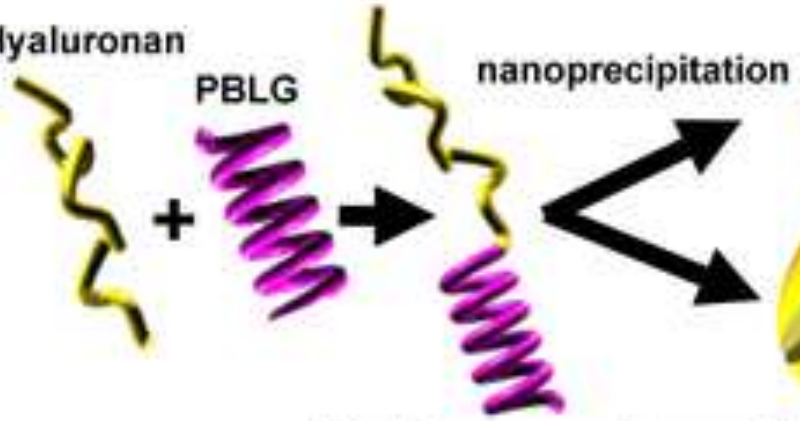

Hyaluronan-based nanoparticles

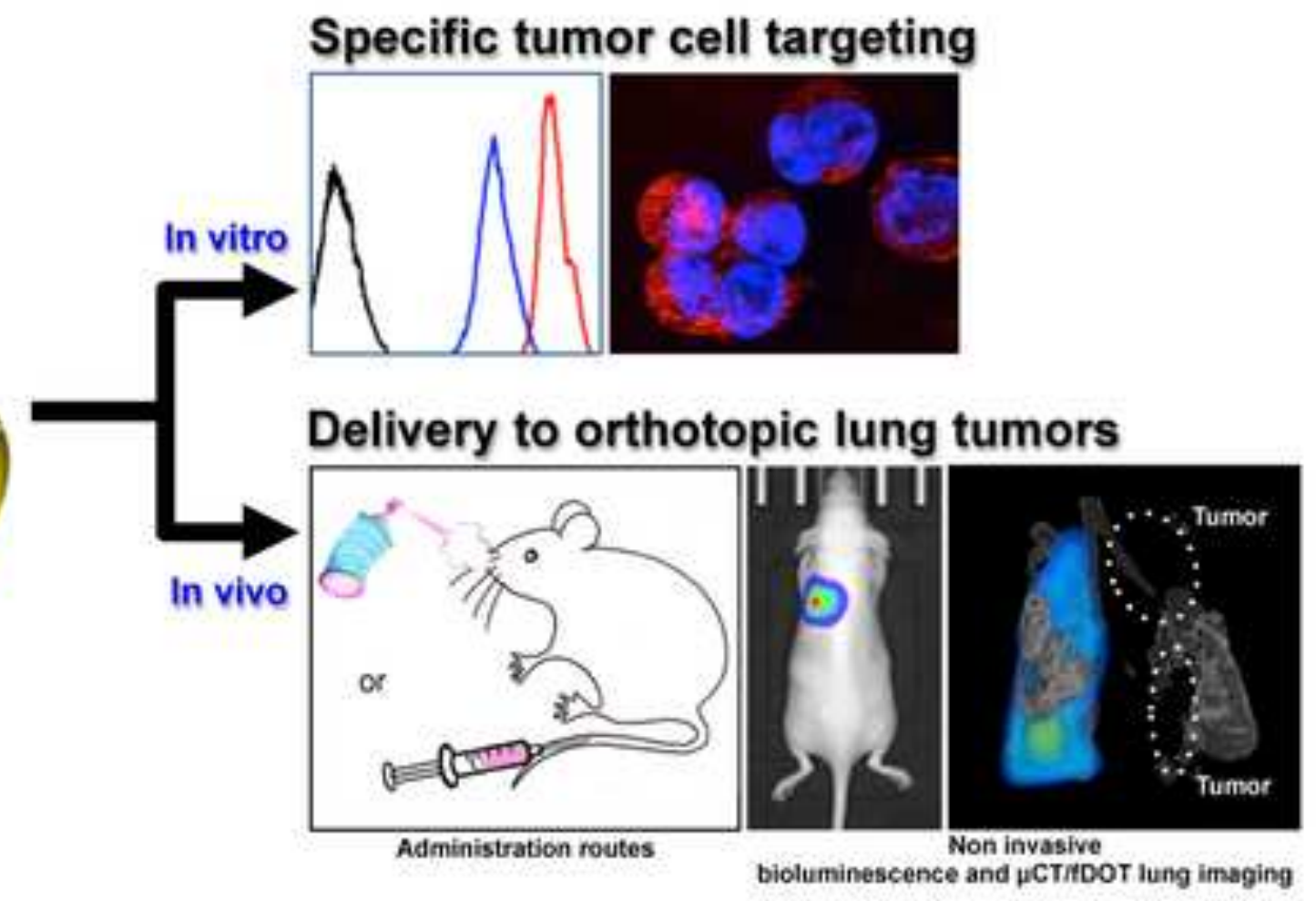




\begin{abstract}
New approaches that are more efficient and able to specifically reach lung tumors are needed. We developed new hyaluronan-based nanoparticles targeting CD44 receptors of two different sizes, 30 and $300 \mathrm{~nm}$, and compared their lung cancer cells targeting efficacy in vitro and in vivo. The nanoparticles cellular uptake was dose-dependent, and specific to hyaluronan receptors, particularly CD44. The binding and internalization differed according to nanoparticle size. In vivo biodistribution studies in two orthotopic lung tumor models showed that intrapulmonary nebulized nanoparticles accumulated in lungs, but not in the tumor nodules. In contrast, despite a significant liver capture, intravenous injection led to a better accumulation of the nanoparticles in the lung tumors compared with the surrounding healthy lung tissues. We demonstrated that the hyaluronanbased nanoparticles size plays significant role in cellular uptake and biodistribution. Small nanoparticles showed active targeting of CD44-overexpressing tumors, suggesting that they could be used as drug-delivery system.
\end{abstract}

\title{
Keywords
}

hyaluronan; polymer nanoparticles; CD44; biodistribution; near-infrared optical imaging; lung cancer 


\section{Introduction}

Lung cancer is the leading cause of cancer-related adult deaths worldwide (1). Non -small cell lung cancers (NSCLCs) account for $\sim 85 \%$ of lung cancers (2). Despite continuous improvements in cancer treatments, the prognosis is poor, and the development of new approaches is urgently needed. In particular, major efforts have been made to generate macromolecular transporters that will deliver the therapeutic molecules specifically to the tumor site, protect them from immediate degradation, allow better control of their spatio-temporal release and thus limit their side effects (3). Polymeric micellar nanostructures have been demonstrated to be powerful tools to address issues of efficacy, specificity, and controlled release of drugs to diseased tissues (4). These structures are able to overcome the body's natural defenses, remain stable for an extended time in circulation, and have tunable physico-chemical properties, allowing control of their permeability and the kinetics of drug release. An active targeting of tumors can be achieved by conjugating a targeting motif that allows the recognition of antigens or overexpressed receptors in targeted tissues (5).

The size of nanoparticles (NPs) is a key factor that affects their in vivo distributions (6, 7). Small NPs $(<10 \mathrm{~nm})$ are quickly cleared by the kidneys and thus have short blood half-lives. Larger NPs are mostly captured by the reticuloendothelial system (RES) (7). In both cases, their half-life depends on their stealthiness. The size of the NPs also affects their ability to flow through poorly structured tumor capillaries, and their endocytosis in mammalian cells $(7,8)$.

The intravenous route is generally used for the administration of NPs in vivo. However, the inhalation of anti-tumor agents may be a local and non-invasive alternative administration route to improve lung cancer treatment because of the large surface area of the lungs for drug absorption and their extensive vasculature with a weak anatomical barrier that does not limit access to the body (9, 10). Direct administration via the airways can increase the amount of locally delivered NPs in lung tumor models (9-11). 
In the present study, we developed glycopolypeptide-based nanocarriers and evaluated their efficacy to target lung tumor cells in vitro and in vivo. These amphiphilic copolymers were combinations of the natural polysaccharide hyaluronan (Hya), known for its relative affinity toward CD44 receptors that are up-regulated on some cancer cells and are involved in tumor growth, progression and metastasis (12), and poly( $\gamma$-benzyl-L-glutamate) (PBLG), a biodegradable polypeptide characterized by an ordered secondary structure ( $\alpha$-helix) $(13,14)$. By varying the hydrophilic/hydrophobic volume ratio of the copolymer and the conditions of particle formation, it was possible to obtain different sizes of NPs, whose we have previously shown the high drug loading capacity (15). Here, we evaluated the behavior of NPs of $30 \mathrm{~nm}(\mathrm{~Np} 30)$ and $300 \mathrm{~nm}$ (Np300) in vitro in lung cancer cells with different levels of CD44. Then, we compared their in vivo biodistribution and tumor targeting capacity after intrapulmonary nebulization or conventional intravenous administration using two orthotopic lung cancer models.

\section{Methods}

\section{Nanoparticle synthesis and characterization}

Synthesis of hyaluronan-b-poly(y-benzyl-L-glutamate) copolymer by 1,3 Huisgen cycloaddition

The strategy used was based on the coupling reaction between poly $(\gamma$-benzyl-L-glutamate) (PBLG) and hyaluronan (Lifecore Biomedical, MW = $5000 \mathrm{~g} / \mathrm{mol}$ ) using a Huisgen 1,3-dipolar cycloaddition click chemistry reaction, as previously described $(16,17)$. The reaction medium was dialyzed 4-5 days against MilliQ water (Spectra/Por®6 MWCO 50 kDa membrane), containing EDTA for the first 2 days, and then lyophilized. Next, the reaction mixture was purified by ultrafiltration to remove all of the unreacted hyaluronan (yield $=60 \%$ ). The resulted copolymer was analyzed by $\mathrm{H}^{1} \mathrm{NMR}$, FTIR and SEC in DMSO to verify the coupling conversion.

\section{Fluorescently labeled nanoparticles}


Hya- $b$-PBLG was labeled by modifying the free amine group at the PBLG end block with the near infrared dye Dy-700 (Life Technologies). Briefly, the diblock copolymer (1 eq.) was dissolved in anhydrous DMSO (50 mg/mL), and triethylamine (1 eq. per acid function) was added. After $5 \mathrm{~min}$ of stirring, the 0.1 equivalent of Dy-700 was added, and the mixture was stirred overnight at room temperature. The resulting product (Hya- $b$-PBLG-Dy-700) was dialyzed against ultrapure water for one day and freeze-dried. A labeling efficiency of $10 \mathrm{~mol} \%$ was measured in DMSO by UV spectroscopy (720 nm) using $\varepsilon=90,000 \mathrm{M}^{-1} . \mathrm{cm}^{-1}$ according to the manufacturer's instructions.

\section{Nanoparticle formulation}

NPs were formulated by a solvent displacement technique, as previously described (15). To obtain labeled fluorescent NPs, a mixture of Hya- $b$-PBLG-Dy700 and Hya- $b$-PBLG copolymer was prepared to obtain $5 \mathrm{~mol} \%$ of labeled copolymer. To obtain different sizes, the originality was to use two types of copolymer with PBLG blocks varying in degree of polymerization (DP 20 and 60) and two different protocols for the nanoprecipitation:

- Slow process: a solution of Hya- $b-\mathrm{PBLG}_{20}$ containing $30 \% \mathrm{w} / \mathrm{w}$ of homo-PBLG $\mathrm{P}_{20}$ was prepared in DMSO $(10 \mathrm{mg} / \mathrm{mL})$ at $50^{\circ} \mathrm{C}$. Using a syringe pump, phosphate buffer $(10 \mathrm{mM}$, $\mathrm{pH}$ 7.4) was added at a flow rate of $70 \mathrm{~mL} / \mathrm{h}$ to reach a final concentration of $1 \mathrm{mg} / \mathrm{mL}$.

- Fast process: Hya-b-PBLG 60 was dissolved in DMSO $\left(10 \mathrm{mg} \cdot \mathrm{mL}^{-1}\right)$ and rapidly mixed with a tenfold volume of phosphate buffer at $50^{\circ} \mathrm{C}$ using a micropipette.

The organic solvent was then removed by dialysis (MWCO $25 \mathrm{kDa}$ ) against water for $24 \mathrm{~h}$, with the medium changed every $4 \mathrm{~h}$. The NPs were then characterized by dynamic light scattering (DLS), transmission electron microscopy (TEM), atomic force microscopy (AFM) and small-angle neutron scattering (SANS).

\section{Cell lines}

The human H322, H358 and A549 NSCLC cell lines were obtained from the American Type Culture Collection (ATCC, Manassas, VA). H358 (H358-Luc) and A549 (A549-Luc) cells stably 
expressing firefly luciferase were obtained from Optimal (Grenoble, France).

\section{Flow cytometry}

CD44 expression levels were determined after cell incubation with FITC-conjugated mouse antiCD44 or control $\mathrm{IgG}_{2 b} \mathrm{~K}$ antibodies (BD Pharmingen, San Diego, CA) for $30 \mathrm{~min}$ at $4{ }^{\circ} \mathrm{C}$. NPs were incubated with cells for $30 \mathrm{~min}$ at $37^{\circ} \mathrm{C}$. For competition assays, cells were previously incubated with 5 or $10 \mathrm{mg} / \mathrm{mL}$ sodium hyaluronan $176-350 \mathrm{kDa}$ (Lifecore Biomedical) for $1 \mathrm{~h}$ at $37^{\circ} \mathrm{C}$ before incubation with $8 \mu \mathrm{g} / \mathrm{mL} \mathrm{NP}$ for $30 \mathrm{~min}$ at $37^{\circ} \mathrm{C}$. The fluorescence intensity was analyzed using flow cytometry LSRII and FCS Express software (BD Biosciences, San Jose, US).

\section{Immunofluorescence and microscopy}

Cells were seeded on glass coverslips coated with $1 \mu \mathrm{g} / \mathrm{mL}$ fibronectin and $1 \%$ bovine serum albumin. After washing, NPs were incubated for $30 \mathrm{~min}$ at $4^{\circ} \mathrm{C}$ or $37^{\circ} \mathrm{C}$. The cells were then washed and fixed in $0.1 \%$ saponin and $2 \%$ paraformaldehyde for $15 \mathrm{~min}$. Nuclei were labeled with DAPI staining. Fluorescence microscopy was carried out using an AxioImager confocal microscope (Carl Zeiss, Jena, Germany).

\section{Orthotopic lung tumor animal models}

Animal experiments studies were approved by the institutional guidelines and by the European Community for the use of experimental animals (authorization to experiment 00393.02). H358-Luc or A549-Luc cells were harvested, washed and resuspended in PBS at $10^{7}$ cells $/ 50 \mu \mathrm{L}$ (H358-Luc) or in PBS/0.5 mM EDTA at $5.10^{6}$ cells/50 $\mu \mathrm{L}$ (A549-Luc). Six-week-old female nude mice (NMRI for H358 tumor model, BalB/c for A549 tumor model) (Janvier, Le Genest, Saint Isle, France) were anesthetized with an intraperitoneal injection of medetomidin $(0.2 \mu \mathrm{g} / \mathrm{g}) / \mathrm{ketamine}(0.1 \mathrm{mg} / \mathrm{g})$, and the cells were inoculated in the lungs with a catheter after orotracheal intubation, as previously described $(11,18,19)$. 
Tumor growth was followed by in vivo bioluminescence imaging (IVIS Kinetic, Perkinelmer) 5 min after the intraperitoneal injection of $150 \mathrm{mg} / \mathrm{kg}$ of Luciferin (Promega, Charbonnières, France), as previously described (20).

\section{Nanoparticle in vivo administration}

Equal quantities of NPs were administered in healthy mice, H358-tumor bearing mice or A549tumor bearing mice after 5-6 weeks of tumor growth. Anesthetized mice (isoflurane/air 4\% for induction and $1.5 \%$ thereafter) were injected intravenously via the tail vein with $200 \mu \mathrm{L}$ of Dy-700 labeled NP ( $8 \mathrm{mg} / \mathrm{mL})$. Intrapulmonary administration of NP was performed using a nebulizing IA1C Microspayer (Penn-Centur, Inc, PA, USA) connected to a FMJ-250-high-pressure syringe (Penn-Centur, Inc) containing $50 \mu \mathrm{L}$ of Dy-700 labeled NPs. After anesthesia with medetomidin/ketamine, the tip of the microsprayer was introduced into the trachea of the animals using a dedicated laryngoscope.

\section{Nanoparticle in vivo biodistribution}

Mice were anesthetized (isoflurane/air 4\% for induction and 2\% thereafter). 2D-fluorescent images and black and white pictures were acquired after intravenous or intrapulmonary administration using a back-thinned CCD camera at $-80^{\circ} \mathrm{C}$ (ORCAII-BT-512 G, Hamamatsu, Massy, France) that was fitted with a long-pass RG 700 filter, as previously described (21). At the end of the experiment, mice were sacrificed and some organs and blood were collected for ex vivo imaging. Semiquantitative data were obtained using the Wasabi ${ }^{\circledR}$ software (Hamamastsu, Massy, France) by drawing regions of interest (ROIs) on the different organs and were expressed as the number of Relative Light Units per pixel per unit of exposure time and relative to the fluorescence signal in the brain. $40 \mu \mathrm{L}$ of blood were sampled from the tail vein before NP administration and at different times after, centrifuged ( $5 \mathrm{~min}$ at $8,000 \mathrm{~g})$ and $10 \mu \mathrm{L}$ of plasma were used for fluorescence imaging. 


\section{Nanoparticle imaging in lung tumors and histology}

Bioluminescence and 2D-fluorescence signals were plotted along a line into the isolated tumorbearing lungs through healthy and tumor tissues according to the distance and quantified using the Zen ${ }^{\circledR}$ software. The ratio between the fluorescent signal in the tumor and the fluorescent signal in the adjacent healthy tissues was calculated.

Confocal microscopy of the lung in frozen sections $(30 \mu \mathrm{m})$ was carried out with a LSM710 LNO confocal microscope (Carl Zeiss, Jena, Germany) using a 60X oil immersion objective. The 702-nm laser intensity was set at 3\% of its maximum intensity. Nuclei were labeled by DAPI.

\section{Statistical analysis}

All data are expressed as the mean \pm SD. Statistical comparisons were made using a Mann-Whitney $U$-test. Two-sided $p$ values $\leq 0.05$ were considered statistically significant. All analyses were performed using the Statview software (Abacus concept, Berkeley, CA, USA).

\section{Results}

\section{Preparation of nanoparticles}

A hydrophilic hyaluronan block was covalently linked by its reductive end to a hydrophobic PBLG (DP 20 and 60) using the 1,3 Huisgen cycloaddition, which combines mild experimental conditions, tolerance of functional groups and nearly quantitative yields (Figure 1A). An original and proper modulation of the copolymer composition and nanoprecipitation conditions (fast and slow processes) allowed the formation of two types of NPs, which had different sizes (Figure 1B). On the one hand, micellar NPs of $30 \mathrm{~nm}(\mathrm{Np30})$ were formulated using a fast nanoprecipitation process (with Hya- $b-\mathrm{PBLG}_{60}$ ). On the other hand, using Hya- $b-\mathrm{PBLG}_{20}$ and a slow process, polydispersed $\mathrm{NP}$ of $300 \mathrm{~nm}(\mathrm{Np300)}$ were generated with a disc-like morphology, in co-existence with small micelles. To reduce the polydispersity and reach a pure NP morphology, $30 \%$ of homo-PBLG 20 was 
added in the initial copolymer solution, according to a recent report (17). The NPs were characterized by DLS, TEM, AFM and SANS (Figure 1C-F).

\section{Nanoparticles bind to NSCLC cells expressing different CD44 levels and are internalized}

The level of expression of CD44 was evaluated by flow cytometry in three different NSCLC cell lines (H322, H358 and A549 cells) (Figure 2A). H322 cells did not express CD44, whereas H358 and A549 cells expressed low and high levels of CD44, respectively. Dose-dependent binding of Np30 and Np300 was observed in the three cell lines, with a higher intensity for A549 cells compared with H322 and H358 cells (Figure 2B). At high NP concentrations (80 and $160 \mu \mathrm{g} / \mathrm{mL}$ ), Np30 binding was 2-3 times higher than Np300 binding in the three cell lines (Figure 2B and supplementary figure S1A). To further evaluate the specificity of NPs toward the hyaluronan cell surface receptors, competitive binding experiments were performed by pretreating the cells with saturating amounts of free hyaluronan (with MW ranging between 175-350 kDa) before NP incubation. Compared with the NP binding without hyaluronan pretreatment, the binding of Np30 on $\mathrm{H} 358$ and A549 cells presented a 50-60\% reduction in the mean fluorescence intensity (MFI) and a 50-70\% reduction when Np300 was used (Figure 2C and supplementary Figure S1B). The inhibitory effect of hyaluronan pretreatment on NP binding increased with hyaluronan concentrations, especially with Np300. The presence of competitive free hyaluronan had no significant effect on Np30 or Np300 binding in H322 cells. The binding of these NPs on A549 and H358 cells were strongly inhibited by an excess of free hyaluronan, thus demonstrating that the hyaluronan cell surface receptors, particularly CD44 receptors, mediated the interaction.

Finally, NP internalization was assessed using confocal microscopy in H358 and A549 cells (Figure 2D). At $4^{\circ} \mathrm{C}$, the internalization process was inhibited and both $\mathrm{Np} 30$ and $\mathrm{Np} 300$ were mostly found on the cell membrane, whereas at $37^{\circ} \mathrm{C}$, punctate labeling was observed for $\mathrm{Np} 30$ and $\mathrm{Np} 300$, which is a hallmark of their intracytoplasmic trafficking after internalization. Cell labeling and internalization were stronger with Np30 than Np300. 


\section{Orthotopic lung tumors}

Orthotopic mouse models of human NSCLCs were established to evaluate the tumor targeting capability of the NPs. These models including the native tumor microenvironnement and angiogenesis are more relevant than the widely used subcutaneous tumors (11). Luciferase-modified human cell lines H358 (H358-Luc cells) or A549 (A549-Luc cells) were inoculated via the airways and noninvasive in vivo bioluminescence was used to monitor the quality of the implantation and to follow orthotopic growth (supplementary Figure S2). Both cell lines led to the development of localized lung tumors $(11,18,19)$. Five weeks after implantation, tumors were well developed and had a strong bioluminescent signal, but the mice did not present any detectable symptoms such as body weight loss or breathing difficulties.

\section{Distribution of the nanoparticles after intravenous injection}

The blood pharmacokinetic of the NPs was measured in healthy mice by fluorescence imaging of plasma samples at different time postinjection (supplementary figure S3). The pharmacokinetic constants were calculated, and the theoretical distribution and elimination half-lives for Np30 (29 and $673 \mathrm{~min}$ ) were higher than for $\mathrm{Np} 300$ (10 and $44 \mathrm{~min}$ for distribution, and $196 \mathrm{~min}$ for elimination), which showed that Np30 had longer circulation times than Np300.

Equal quantities of Dy-700-labeled Np30 or Np300 were injected intravenously in healthy or tumor bearing mice, and their biodistributions were assessed at multiple time points using 2D-FRI optical imaging. Liver uptake was fast and strong for both NPs in healthy and tumor-bearing mice, and the fluorescent signal remained stable over $24 \mathrm{~h}$ (Figure 3A). At this time, the mice were euthanized, and the collected organs were subjected to ex vivo bioluminescence and fluorescence imaging (Figure 3B-D). For both NPs, the strongest fluorescence signals were measured in the liver and spleen, which highlighted their hepatic elimination. The Np30 and Np300 biodistribution patterns were similar in mice bearing tumors or not. The fluorescence of the lungs ex vivo was assessed to 
compare the accumulation of NPs in healthy $v s$ tumor-bearing lungs. The Np30 fluorescent signal was slightly higher in H358 tumor-bearing lungs and was 2 times higher in A549 tumor-bearing lungs than in healthy lungs (figure 4A). In contrast, the Np300 fluorescent signal in H358 tumorbearing lungs was not significantly different from the one in healthy lungs and was slightly decreased in A549 tumor-bearing lungs compared with healthy lungs.

To evaluate whether the NPs accumulated preferentially in the tumor or healthy part of the lungs, the bioluminescence and fluorescence signals were overlaid, and their respective intensities plotted along a line (Figure 4B-C). We observed that the maximum of Np30 fluorescent signal co-localized with the tumor bioluminescent signal. The fluorescent signal was stronger in the tumor than in the surrounding healthy tissue, as evidenced by the fluorescence ratios of $1.61 \pm 0.85$ and $1.38 \pm 0.29$ in H358 and A549 tumor-bearing lungs respectively. By contrast, no tumor accumulation was detected for Np300. Indeed, the fluorescent signal was not significantly higher in H358 (1.04 \pm 0.38$)$ or A549 $(1.35 \pm 0.43)$ tumors than in the adjacent healthy tissues. Using confocal microscopy on lung sections, we observed weak Np30- and Np300-related fluorescent signals in healthy tissue and in H358 and A549 tumors (figure 4D), but these signals were too sparse to be quantified.

Altogether, these results showed that after intravenous injection, both $\mathrm{Np30}$ and Np300 were strongly retained by the liver. However, a very small proportion reached the lungs. Np30 accumulated more strongly in the A549 and H358 tumor-bearing lungs, as compared with normal lungs (Figure 4A), and the fluorescence signal correlated well with the tumor bioluminescent signal. When Np300 was used, no particular accumulation in the invaded lung could be measured, and the NPs that were present in the lung were dispersed equally in the tumor and healthy areas.

\section{Distribution of the nanoparticles after intrapulmonary administration}

The NP biodistributions after the intrapulmonary administration of equal quantities of Dy-700labeled Np30 or Np300 were evaluated at multiple time points in healthy mice and H358- or A549tumor bearing mice. Representative images are shown in Figure 5A. Both Np30 and Np300 signals 
were detected in the lungs immediately after administration in healthy and tumor-bearing mice, and they remained stable over $24 \mathrm{~h}$ (Figure $5 \mathrm{~A}$ ). After quantification in the extracted organs at $24 \mathrm{~h}$ (figure 5B), the fluorescence signal was 15 to 20 times higher in the lungs than in other organs, without significant differences between healthy and H358- or A549-tumor bearing mice (figure 5CD). No significant fluorescence signal was observed in the other organs, including liver or kidney, which confirmed a strong retention of the NPs in the airways.

The levels of nebulized Np30 or Np300 in tumor-bearing lungs were not significantly different from those in healthy lungs (figure 6A), which indicates that the NPs did not accumulate in lung tumors after intrapulmonary administration. Bioluminescence and fluorescence signal overlays indicated that neither Np30 nor Np300 co-localized with the tumors (Figure 6B-C). The ratios between the fluorescent signals in tumors and in adjacent healthy tissues showed that the NP signals were higher in healthy tissue than in $\mathrm{H} 358$ or A549 tumors for both $\mathrm{Np30}(0.59 \pm 0.44$ and $0.53 \pm 0.11$, respectively) and $\mathrm{Np} 300(0.50 \pm 0.04$ and $0.91 \pm 0.50$, respectively $)$. The results were confirmed by confocal microscopy (figure 6D). Stronger fluorescence signals were observed in healthy tissue adjacent to the tumors than in lung tumors, thus illustrating that NPs did not reach lung tumor cells after intrapulmonary administration. In addition, the NPs' access to the invaded areas of the lung was reduced by airway obstruction resulting from the presence of tumors. These animals were thus imaged with microCT and 3D-fluorescence tomography, in addition to bioluminescence. Examples of mice with well-established H358 lung tumors are shown in supplementary figure S4. From microCT acquisitions we reconstructed and extracted 3D images that represent the presence of air in the lungs. As can be seen on these images, the presence of tumors strongly reduced the volume of air in the lungs, and no NPs fluorescence signal was found in these "empty" regions of microCT in either Np30- or Np300-treated animals. 


\section{Discussion}

We generated hyaluronan-based NPs with two different sizes: nanospheres (Np30, $30 \mathrm{~nm}$ diameter) and disk-like NPs (Np300, $300 \mathrm{~nm}$ diameter). Regarding the large difference of size between the two types of NPs, it is expected that the differences in the biological behavior are mostly related to the size difference rather than the shape. We studied the influence of the size on the hyaluronanmediated CD44-dependent cellular binding in vitro and on their biodistribution profiles after intravenous or nebulization administration on mice bearing orthotopic lung tumors.

Both Np30 and Np300 are made with low molecular weight hyaluronan (5 kDa). Hyaluronan is the natural ligand of the CD44 receptors and is overexpressed in many tumors (12). In addition to CD44, hyaluronan interacts with other cell surface receptors such as the receptor for hyaluronanmediated motility (RHAMM), the HARE receptor, Toll-Like receptors-2 and -4 (TLR-2 and TLR4) and LYVE-1 $(22,23)$. Our results showed the high expression of CD44 in A549 cells and its low expression in H358 cells. RHAMM is not expressed in A549 cells (24). The HARE receptor is mainly expressed in the sinusoidal endothelial cells of liver, spleen, and lymph nodes (22), LYVE-1 in lymphatic tissue, and TLR-2/4 expressed in dendritic cells recognize hyaluronan during inflammatory events (25). These data indicated that CD44 should be the main hyaluronan receptor for mediating Np30 and Np300 binding on A549 and H358 tumor cells but that it is poorly implicated in H322 cells. In agreement, we observed that both NPs showed a dose-dependent binding on the cells, with A549 being more intensively stained than H358 and H322 cells. Competition assays with free hyaluronan showed a significant reduction of NPs binding corroborating the specificity to hyaluronan receptors.

In order to compare the cellular uptake of NPs, we used Np30 and Np300 copolymer concentrations allowing equivalent signals of fluorescence and surface areas of binding. Np30 showed a higher 
cellular binding and internalization than Np300, in agreement with studies that showed NPs with a 20-25 nm radius achieved effective cellular uptake (8). The use of clathrin-mediated entry to internalize particles with sizes ranging from 10 to $300 \mathrm{~nm}$ has been reported (8). It has also been proposed that negatively charged NP, including hyaluronan-based NP, would be mostly internalized owing to caveolae-mediated endocytosis $(8,26)$. Internalization of nanodisks could also be slower than that of nanospheres, which could explain the difference between the Np30 and Np300 uptake profiles in NSCLC cells. Further experiments are needed to clarify if the cellular uptake differences for $\mathrm{Np} 30$ and Np300 are mainly due to size or shape.

The behavior of NPs in vivo is strongly affected by their interaction with blood components and by biological factors, such as tumor presence, size, and location, angiogenesis, inflammation, and stroma thickness, composition, and rigidity. These factors will dramatically modify NPs surface properties in a highly dynamic and rapidly changing manner $(7,27)$. These aspects are difficult to investigate since it is very often impossible to recover the NPs in vivo to study their actual composition.

Despite a longer circulation time for Np30, both $\mathrm{Np} 30$ and Np300 were rapidly taken up by the liver after intravenous injection. This observation is in accordance with the literature describing a rapid clearance by the kidneys for the small particles $(<10 \mathrm{~nm})$, whereas larger particles are more efficiently captured by the RES and sequestered by the liver because of opsonization and protein association on the NP surface (7, 28-30). In addition, Np30 and Np300 are negatively charged, which is also a key factor that drives NP behavior, promoting recognition by the RES and uptake by the liver $(7,31)$.

Semiquantitative measure of fluorescence signal (32) showed that Np30 was significantly higher in A549 tumor-bearing lungs than in healthy lungs. This could be in part explained by the enhanced permeability and retention (EPR) effect: the tumor vasculature is irregular, leaky, and dilated, and the vascular endothelial cells are poorly aligned with large fenestrations, resulting in the increased 
leakage of nanocarriers from the circulatory system into the tumor tissue (33). Accordingly, the longer circulation time measured for $\mathrm{Np30}$, compared with $\mathrm{Np300}$, could explain the higher accumulation of Np30 in tumor-bearing lungs. However, Np30 accumulation was significantly higher in the CD44-overexpressing A549 tumor cells than in H358 low-expressing cells or in healthy tissue, which suggests that CD44 receptor active targeting could play a role in Np30 retention in lung tumors, although this has yet to be formally established.

The intrapulmonary delivery of antitumoral agents might enhance their amount at the targeted lung cancer site, thereby improving NP accumulation in tumors and the antitumoral effect $(9,11)$. The nebulized NPs presented a similar and very strong accumulation in healthy, H358 or A549 lungs, and the fluorescent signal remained stable for at least $72 \mathrm{~h}$ (data not shown). Moreover, no fluorescence signal was observed in the other organs, in contrast to ultra-small NP $(<6 \mathrm{~nm})$, which showed a passage from the airways to the blood after nebulization and a renal elimination (11). The larger size of $\mathrm{Np} 30$ and $\mathrm{Np} 300$ could explain their long retention in the lungs without diffusion into the blood stream. As a consequence, this would also prevent such NPs from "returning" to the tumors via the blood circulation and the EPR effect.

The semiquantitative measure of fluorescence signals (32) of both NPs were not different between tumor-bearing and healthy lungs and confocal microscopy demonstrated that NPs did not reach tumor tissue. The bronchial obstruction due to the presence of tumor nodules might prevent the NP spray from penetrating the tumor, and thus might accumulate in healthy tissue.

In summary, we demonstrated that the size of hyaluronan-based NPs plays a critical role in cellular uptake, in correlation with the CD44 level. In the NSCLC cells, NP of $30 \mathrm{~nm}$ were bound and internalized more efficiently than were the $300 \mathrm{~nm}$ NP. In vivo studies showed that direct administration of these NPs into the airways failed to increase their uptake by the tumors. However, 
their intravenous injection showed a preferential and possibly active targeting of CD44-positive tumors for $30 \mathrm{~nm}$ NPs that could not be documented for the $300 \mathrm{~nm}$ NPs. 


\section{References}

1. Siegel R, Naishadham D, Jemal A. Cancer statistics, 2012. CA: a cancer journal for clinicians. 2012;62(1):10-29.

2. Pisters KM, Evans WK, Azzoli CG, Kris MG, Smith CA, Desch CE, et al. Cancer Care Ontario and American Society of Clinical Oncology adjuvant chemotherapy and adjuvant radiation therapy for stages I-IIIA resectable non small-cell lung cancer guideline. Journal of clinical oncology : official journal of the American Society of Clinical Oncology. 2007;25(34):5506-18.

3. Peer D, Karp JM, Hong S, Farokhzad OC, Margalit R, Langer R. Nanocarriers as an emerging platform for cancer therapy. Nature nanotechnology. 2007;2(12):751-60.

4. De Oliveira H, Thevenot J, Lecommandoux S. Smart polymersomes for therapy and diagnosis: fast progress toward multifunctional biomimetic nanomedicines. Wiley interdisciplinary reviews Nanomedicine and nanobiotechnology. 2012;4(5):525-46.

5. Byrne JD, Betancourt T, Brannon-Peppas L. Active targeting schemes for nanoparticle systems in cancer therapeutics. Advanced drug delivery reviews. 2008;60(15):1615-26.

6. Alexis F, Pridgen E, Molnar LK, Farokhzad OC. Factors affecting the clearance and biodistribution of polymeric nanoparticles. Molecular pharmaceutics. 2008;5(4):505-15.

7. Dufort S, Sancey L, Coll JL. Physico-chemical parameters that govern nanoparticles fate also dictate rules for their molecular evolution. Adv Drug Deliv Rev. 2012;64(2):179-89.

8. Akinc A, Battaglia G. Exploiting endocytosis for nanomedicines. Cold Spring Harbor perspectives in biology. 2013;5(11):a016980.

9. Gagnadoux F, Hureaux J, Vecellio L, Urban T, Le Pape A, Valo I, et al. Aerosolized chemotherapy. Journal of aerosol medicine and pulmonary drug delivery. 2008;21(1):61-70.

10. Azarmi S, Roa WH, Lobenberg R. Targeted delivery of nanoparticles for the treatment of lung diseases. Adv Drug Deliv Rev. 2008;60(8):863-75. 
11. Dufort S, Bianchi A, Henry M, Lux F, Le Duc G, Josserand V, et al. Nebulized gadoliniumbased nanoparticles: a theranostic approach for lung tumor imaging and radiosensitization. Small. 2015;11(2):215-21.

12. Platt VM, Szoka FC, Jr. Anticancer therapeutics: targeting macromolecules and nanocarriers to hyaluronan or CD44, a hyaluronan receptor. Molecular pharmaceutics. 2008;5(4):474-86.

13. Crespo JS, Lecommandoux S, Borsali R, Klok HA, Soldi V. Small angle neutron scattering from diblock copolymer poly(styrene-d8)-b-poly(g-benzyl-L-glutamate) solutions : Rod-Coil to Coil-Coil transition. Macromolecules. 2003;36(4):1253-6.

14. Lecommandoux S, Achard MF, Langenwalter JF, Klok HA. Self-Assembly of Rod-Coil Diblock Oligomers Based on a -Helical Peptides. Macromolecules. 2001;34(26):9100-11.

15. Kakkar D, Mazzaferro S, Thevenot J, Schatz C, Bhatt A, Dwarakanath BS, et al. Amphiphilic PEO-b-PBLG diblock and PBLG-b-PEO-b-PBLG triblock copolymer based nanoparticles: doxorubicin loading and in vitro evaluation. Macromol Biosci. 2015;15(1):124-37.

16. Upadhyay KK, Le Meins JF, Misra A, Voisin P, Bouchaud V, Ibarboure E, et al. Biomimetic doxorubicin loaded polymersomes from hyaluronan-block-poly(gamma-benzyl glutamate) copolymers. Biomacromolecules. 2009;10(10):2802-8.

17. Bonduelle C, Mazzaferro S, Huang J, Lambert O, Heise A, Lecommandoux S. Synthesis and self-assembly of branched glycopolypeptides: effect of topology and conformation. Faraday discussions. 2013;166:137-50.

18. Guilleminault L, Azzopardi N, Arnoult C, Sobilo J, Herve V, Montharu J, et al. Fate of inhaled monoclonal antibodies after the deposition of aerosolized particles in the respiratory system. Journal of controlled release : official journal of the Controlled Release Society. 2014;196:344-54.

19. Bianchi A, Dufort S, Lux F, Fortin PY, Tassali N, Tillement O, et al. Targeting and in vivo imaging of non-small-cell lung cancer using nebulized multimodal contrast agents. Proc Natl Acad Sci U S A. 2014;111(25):9247-52. 
20. Keramidas M, Josserand V, Righini CA, Wenk C, Faure C, Coll JL. Intraoperative nearinfrared image-guided surgery for peritoneal carcinomatosis in a preclinical experimental model. The British journal of surgery. 2010;97(5):737-43.

21. Bianchi A, Dufort S, Lux F, Courtois A, Tillement O, Coll JL, et al. Quantitative biodistribution and pharmacokinetics of multimodal gadolinium-based nanoparticles for lungs using ultrashort TE MRI. MAGMA. 2014;27(4):303-16.

22. Qhattal HS, Liu X. Characterization of CD44-mediated cancer cell uptake and intracellular distribution of hyaluronan-grafted liposomes. Molecular pharmaceutics. 2011;8(4):1233-46.

23. Arpicco S, De Rosa G, Fattal E. Lipid-Based Nanovectors for Targeting of CD44Overexpressing Tumor Cells. Journal of drug delivery. 2013;2013:860780.

24. Agut W, Agnaou R, Lecommandoux S, Taton D. Synthesis of Block Copolypeptides by Click Chemistry. Macromolecular Rapid Communications. 2008;29(12-13):1147-55.

25. Toole BP. Hyaluronan-CD44 Interactions in Cancer: Paradoxes and Possibilities. Clin Cancer Res. 2009;15(24):7462-8.

26. Dufay Wojcicki A, Hillaireau H, Nascimento TL, Arpicco S, Taverna M, Ribes S, et al. Hyaluronic acid-bearing lipoplexes: physico-chemical characterization and in vitro targeting of the CD44 receptor. Journal of controlled release : official journal of the Controlled Release Society. 2012;162(3):545-52.

27. Fang J, Nakamura H, Maeda H. The EPR effect: Unique features of tumor blood vessels for drug delivery, factors involved, and limitations and augmentation of the effect. Adv Drug Deliv Rev. 2011;63(3):136-51.

28. Choi HS, Ashitate Y, Lee JH, Kim SH, Matsui A, Insin N, et al. Rapid translocation of nanoparticles from the lung airspaces to the body. Nat Biotechnol. 2010;28(12):1300-3.

29. Choi HS, Liu W, Liu F, Nasr K, Misra P, Bawendi MG, et al. Design considerations for tumour-targeted nanoparticles. Nat Nanotechnol. 2010;5(1):42-7. 
30. $\mathrm{Yu} \mathrm{M,} \mathrm{Zheng} \mathrm{J.} \mathrm{Clearance} \mathrm{Pathways} \mathrm{and} \mathrm{Tumor} \mathrm{Targeting} \mathrm{of} \mathrm{Imaging} \mathrm{Nanoparticles.} \mathrm{ACS}$ Nano. 2015;9(7):6655-74.

31. Xiao K, Li Y, Luo J, Lee JS, Xiao W, Gonik AM, et al. The effect of surface charge on in vivo biodistribution of PEG-oligocholic acid based micellar nanoparticles. Biomaterials. 2011;32(13):3435-46.

32. Dufort S, Sancey L, Wenk C, Josserand V, Coll JL. Optical small animal imaging in the drug discovery process. Biochim Biophys Acta. 2010;1798(12):2266-73.

33. Kobayashi H, Watanabe R, Choyke PL. Improving conventional enhanced permeability and retention (EPR) effects; what is the appropriate target? Theranostics. 2013;4(1):81-9. 


\section{Figure legends}

Figure 1: Synthesis and self-assembly of the hyaluronan-b-PBLG copolymer.

A: Chemical pathway toward hyaluronan- $b$-PBLG copolymer and its labeling with the fluorescent dye Dy-700. B: Elaboration of bock copolymer NP through a fast or slow nanoprecipitation protocol. C: Characterization of block copolymer NP by static and dynamic light scattering. $\mathrm{R}_{\mathrm{H}}$ : hydrodynamic radius, PDI: polydispersity index, $\mathrm{M}_{\mathrm{w}}$ : weight-average molecular weight, $\mathrm{N}_{\mathrm{agg}}$ : aggregation number (=number of copolymer chains in a NP); Rg,z: z-average root-mean-square radius of gyration. D: intensity-weighted size distribution of NP obtained by DLS analysis at a detection angle of $90^{\circ}$. E, F: AFM pictures of Np30 (E, $\left.500 \times 500 \mathrm{~nm}\right)$ and Np300 (F, 3 x $\left.3 \mu \mathrm{m}\right)$.

Figure 2: In vitro uptake of nanoparticles by NSCLC cells.

A: Flow cytometry histograms shows cells stained with FITC-CD44 antibodies (blue curve), FITC$\operatorname{IgG}_{2 b}$ (red curve), or without antibodies (black curve). B: Concentration-dependent binding of Dy700-labeled Np30 (left) and Np300 (right) after $30 \mathrm{~min}$ at $37^{\circ} \mathrm{C}$ in $\mathrm{H} 322, \mathrm{H} 358$ and $\mathrm{A} 549$ cells. The results are expressed as the mean fluorescence intensity (MFI) \pm SD normalized against untreated cells $(\mathrm{n}=3)$. C: Np30 (left) and Np300 (right) $(8 \mu \mathrm{g} / \mathrm{mL})$ binding to H322, H358, and A549 cells with or without 5 or $10 \mathrm{mg} / \mathrm{mL}$ hyaluronan (HYA) pretreatment. The results are expressed as the percentage of the $\mathrm{MFI} \pm \mathrm{SD}$ normalized against cells without HYA pretreatment $(100 \%)(\mathrm{n}=3)$. ${ }^{*} p \leq 0.05$, compared with NP treatment without HYA pretreatment. D: Confocal microscopy images display cellular binding and internalization of $160 \mu \mathrm{g} / \mathrm{mL}$ NPs in H358 and A549 cells after 30 min at $4{ }^{\circ} \mathrm{C}$ and $37^{\circ} \mathrm{C}$. Blue channel: DAPI-stained nuclei; red channel: Dy-700-labeled NPs; magnification: $60 \mathrm{X}$.

Figure 3: Biodistribution of fluorescent nanoparticles after intravenous administration in orthotopic lung tumors. 
In vivo biodistribution of Dy-700-labeled NPs in healthy, H358-Luc, or A549-Luc lung tumorbearing mice were studied. A: Fluorescence images (500 ms integration time) were recorded at different times after injection (min-max: frontal 1745-30629; back 1745-7498). An example of the biodistribution in healthy mice treated with Np30 and Np300 is presented (for each NP: upper line, dorsal view of the mouse; lower line, ventral view of the mouse). B: Fluorescence images were performed on isolated organs $24 \mathrm{~h}$ after injection. An example of organs extracted from a healthy mouse treated with Np30 is presented. C-D: ROI are defined on the extracted organs to semiquantify the amount of photons detected per pixel after a $200 \mathrm{~ms}$ exposure. The results in each

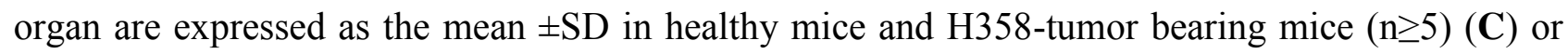
A549-tumor bearing mice ( $\mathrm{n} \geq 4)$ (D).

Figure 4: Colocalization of bioluminescence and fluorescence in orthotopic lung tumors after intravenous administration of nanoparticles.

A: ROI were defined in the extracted lungs to semi-quantify the amount of photons detected per pixel after $200 \mathrm{~ms}$ exposure after intravenous injection of NPs. The results are expressed as the mean $\pm \mathrm{SD}$ and as the rate of signal in healthy mice. H358-tumor bearing mice $\mathrm{n} \geq 5$, A549-tumor bearing mice $\mathrm{n} \geq 4$. ${ }^{*} p \leq 0.05$, compared to healthy mice. B-C: Bioluminescence and fluorescence imaging was performed on isolated lungs, and showed a colocalization between the H358-Luc (B) or A549-Luc (C) lung tumors (bioluminescence, in red) and the fluorescent NP (fluorescence, in green). Bioluminescent and fluorescent signals are plotted according to the distance in lungs (arrows). D: Lung tumors were extracted and cryosectioned before confocal microscopy observation. In blue: DAPI-stained nuclei; in red: Dy-700 signal; in white and black: superimposed visible light images. Np30 or Np300 signals in adjacent healthy tissue (a and d), H358 tumors (b and e) or A549 tumors (c and f). Magnification: 60X. 
Figure 5: Biodistribution of fluorescent nanoparticles after nebulization in orthotopic lung tumor.

In vivo biodistribution of Dy-700-labeled NPs in healthy, H358-Luc, or A549-Luc lung tumorbearing mice were studied. A: Fluorescence images (500 ms integration time) were recorded at different times after nebulization (min-max: 1774-6508). An example of biodistribution in A549 tumor-bearing mice treated with Np30 and Np300 is presented (for each NP: upper line, dorsal view of the mouse; lower line, ventral view of the mouse). B: Fluorescence images were obtained of isolated organs $24 \mathrm{~h}$ after nebulization. An example of organs extracted from an A549 tumor-bearing mouse treated with Np30 is presented. C-D: ROI were then defined on the extracted organs to semiquantify the amount of photons detected per pixel after a $200 \mathrm{~ms}$ exposure. The results in each organ are expressed as the mean $\pm \mathrm{SD}$ in healthy mice and H358-tumor bearing mice $(\mathrm{n} \geq 3)(\mathbf{C})$ or A549-tumor bearing mice $(\mathrm{n}>3)(\mathbf{D})$

Figure 6: Colocalization of bioluminescence and fluorescence in orthotopic lung tumors after nebulization of nanoparticles.

A: ROI were defined in the extracted lungs to semi-quantify the amount of photons detected per pixel after $200 \mathrm{~ms}$ exposure after intrapulmonary administration of NPs. The results are expressed as the mean $\pm \mathrm{SD}$ and as rate of signal in healthy mice ( $\mathrm{n} \geq 3$ mice per group). B-C: Bioluminescence in H358-Luc (B) or A549-Luc (C) lung tumors (bioluminescence, in red) and fluorescence of Np30 or Np300 (fluorescence, in green) imaging were performed on isolated lungs. Bioluminescent and fluorescent signals are plotted according to the distance in lungs (arrows). D: Lungs tumors were extracted and cryosectioned before confocal microscopy observation. In blue: DAPI-stained nuclei; in red: Dy-700 signal; in white and black: superimposed visible light images. Np30 or Np300 signals in adjacent healthy tissue (a and d), H358 tumors (b and e) or A549 tumors (c and f). Magnification: 60X. 
Click here to download high resolution image

\section{Figure 1}

A

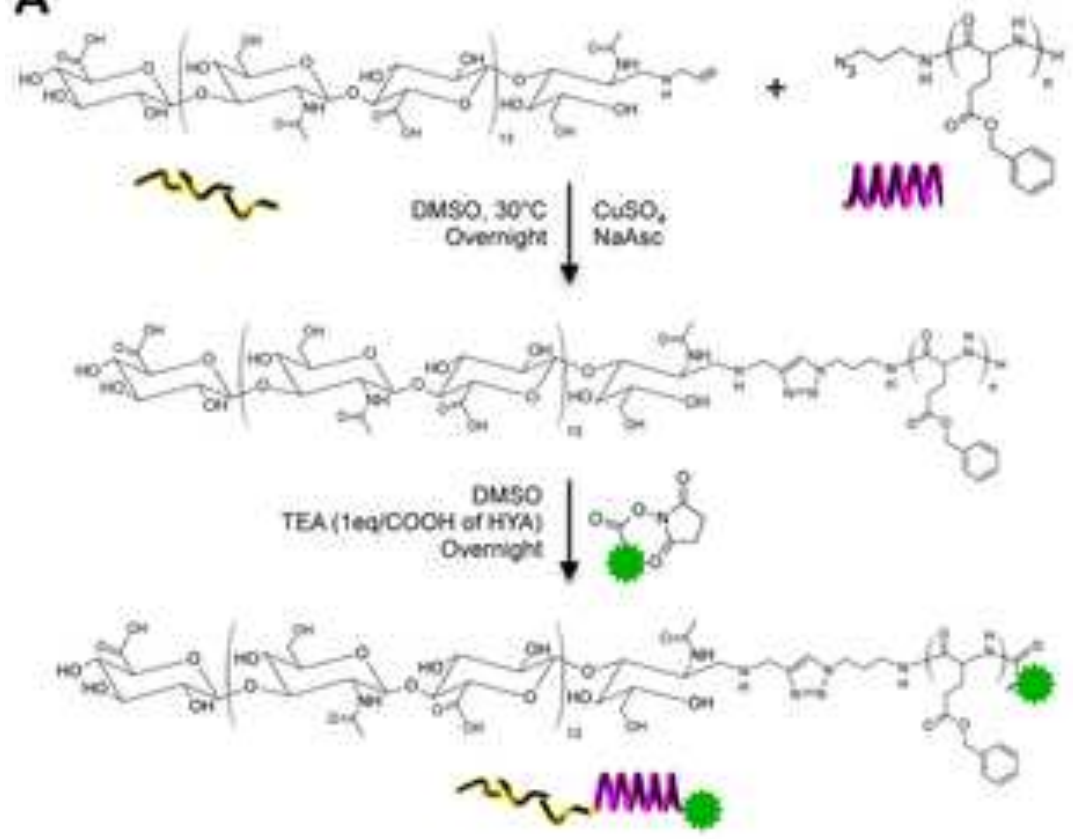

B

NP300 $\mathrm{Hya}_{5}-b-\mathrm{PBLG}_{20}+30 \%$ homo PBLG 20 Slow Nanoprecipitation

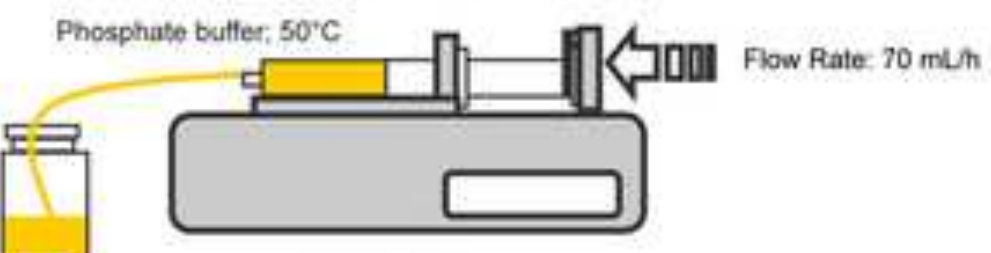

Copolymer in DMSO; $50^{\circ} \mathrm{C}$

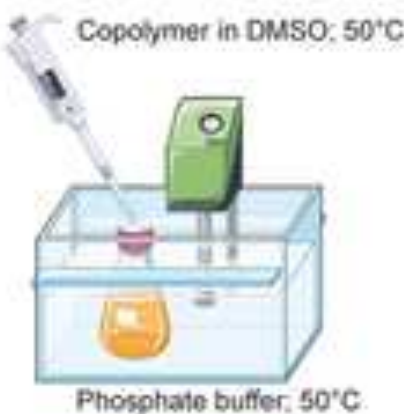

Fast Nanoprecipitation
C

\begin{tabular}{|c|c|c|}
\hline & NP30 & NP300 \\
\hline$R_{4}(n m)$ & 18 & 134 \\
\hline PDI & 0.21 & 0.15 \\
\hline $\mathrm{Mw}(9 / \mathrm{mol})$ & $1.32 E+06$ & $5,15 E+90$ \\
\hline Nagg & 7.37E+01 & $5.36 E+04$ \\
\hline $\mathrm{R}_{9 . z}(\mathrm{~nm})$ & NA & 130 \\
\hline
\end{tabular}

D

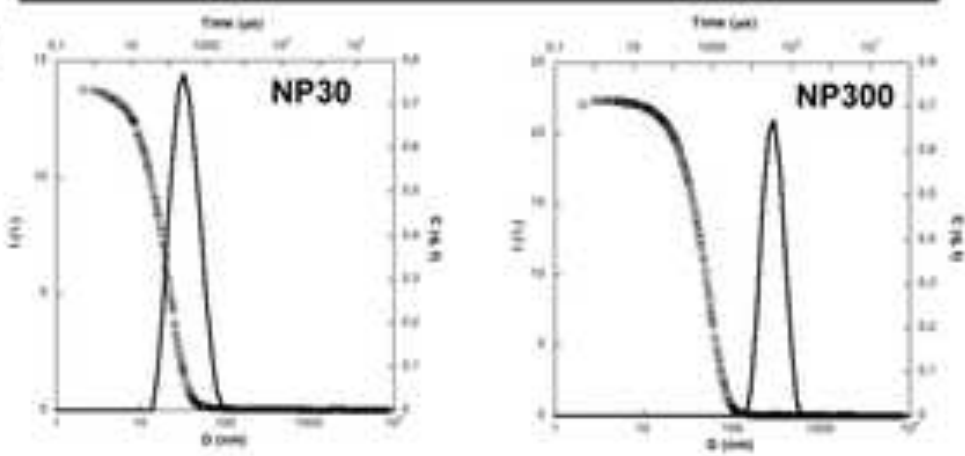

E

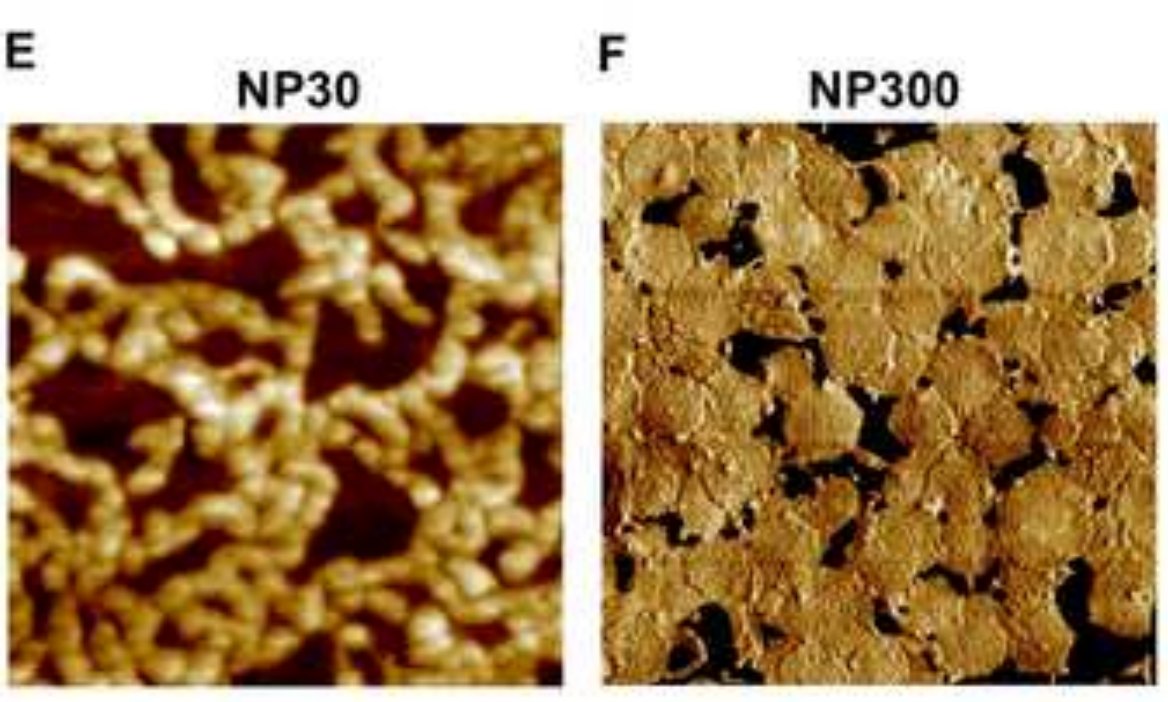

$\mathbf{F}$

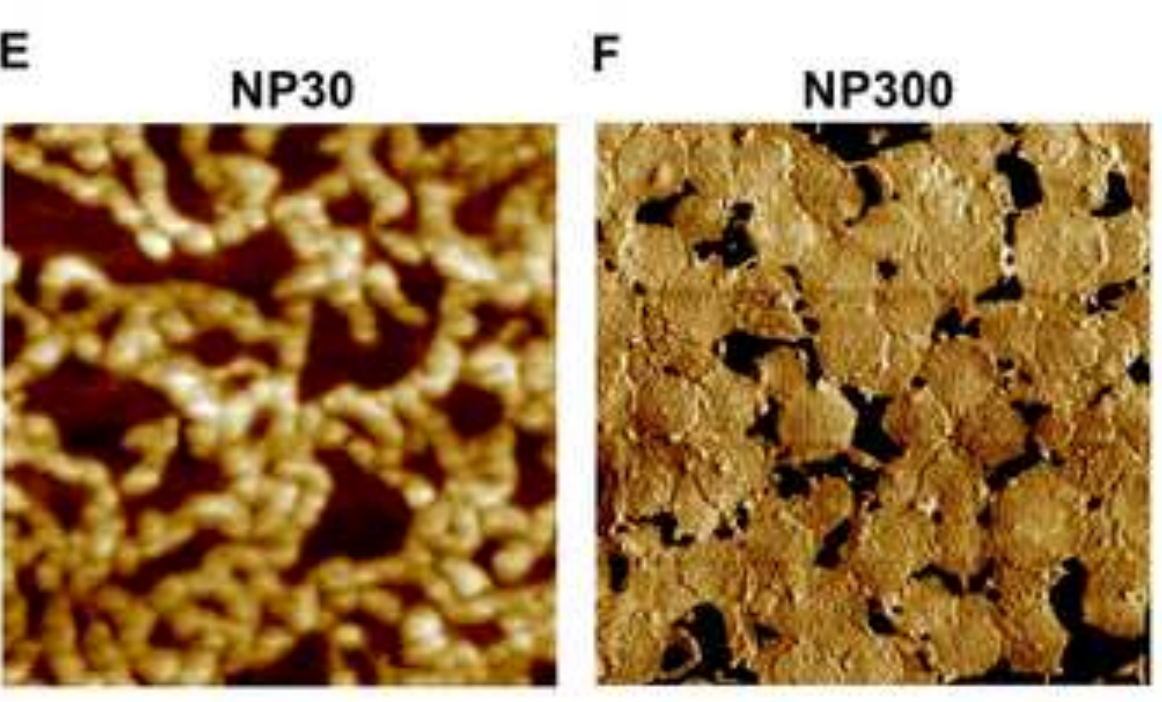


Figure 2

A

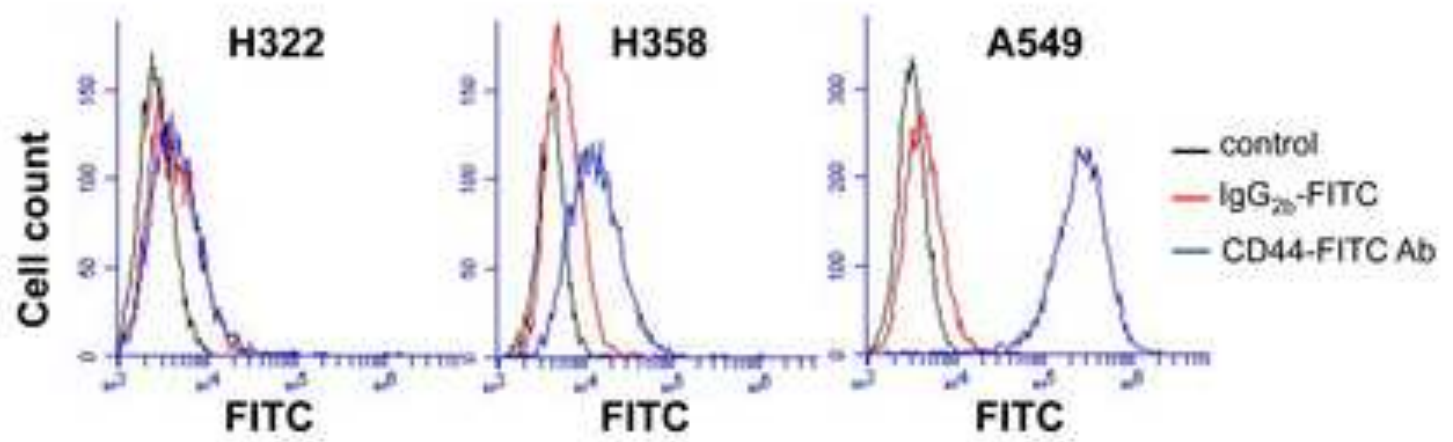

B
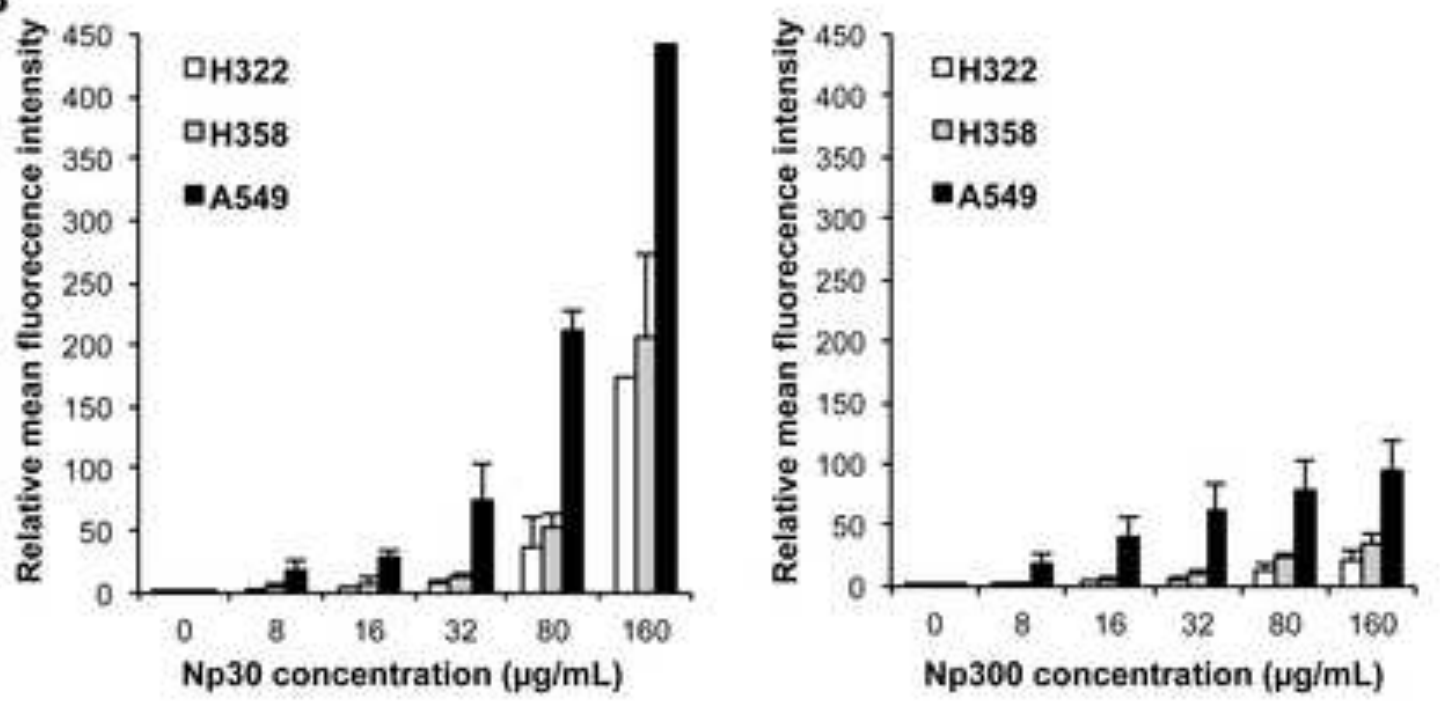

C

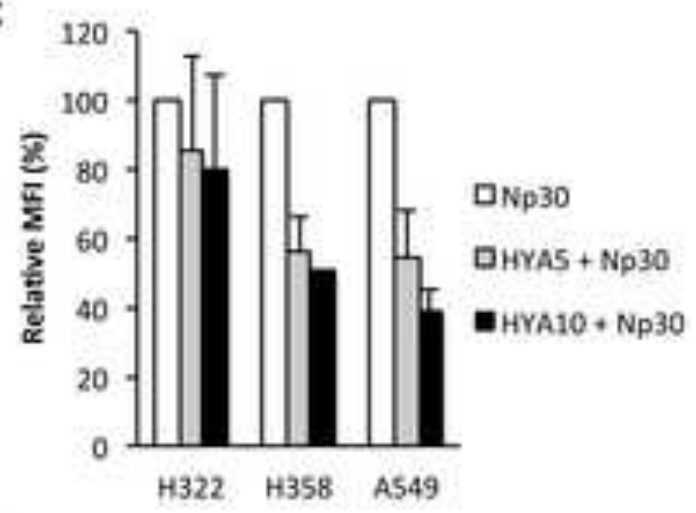

D

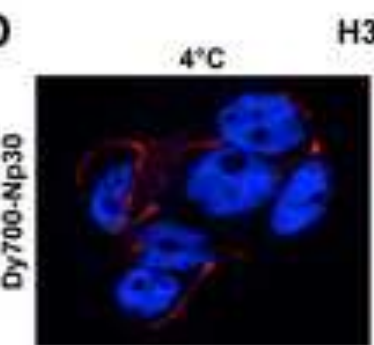

H358

$37^{*} \mathrm{C}$
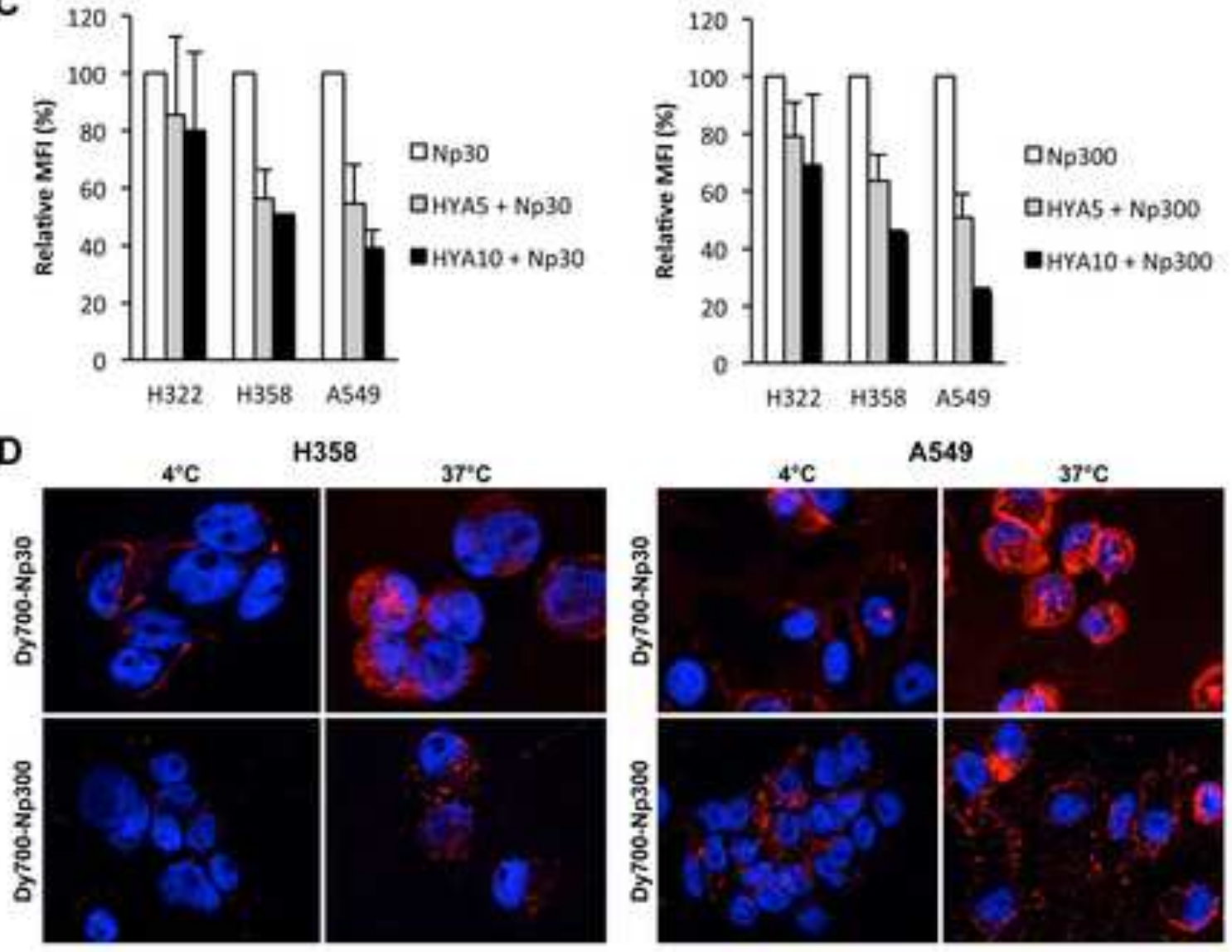
Figure 3

Intravenous administration

A
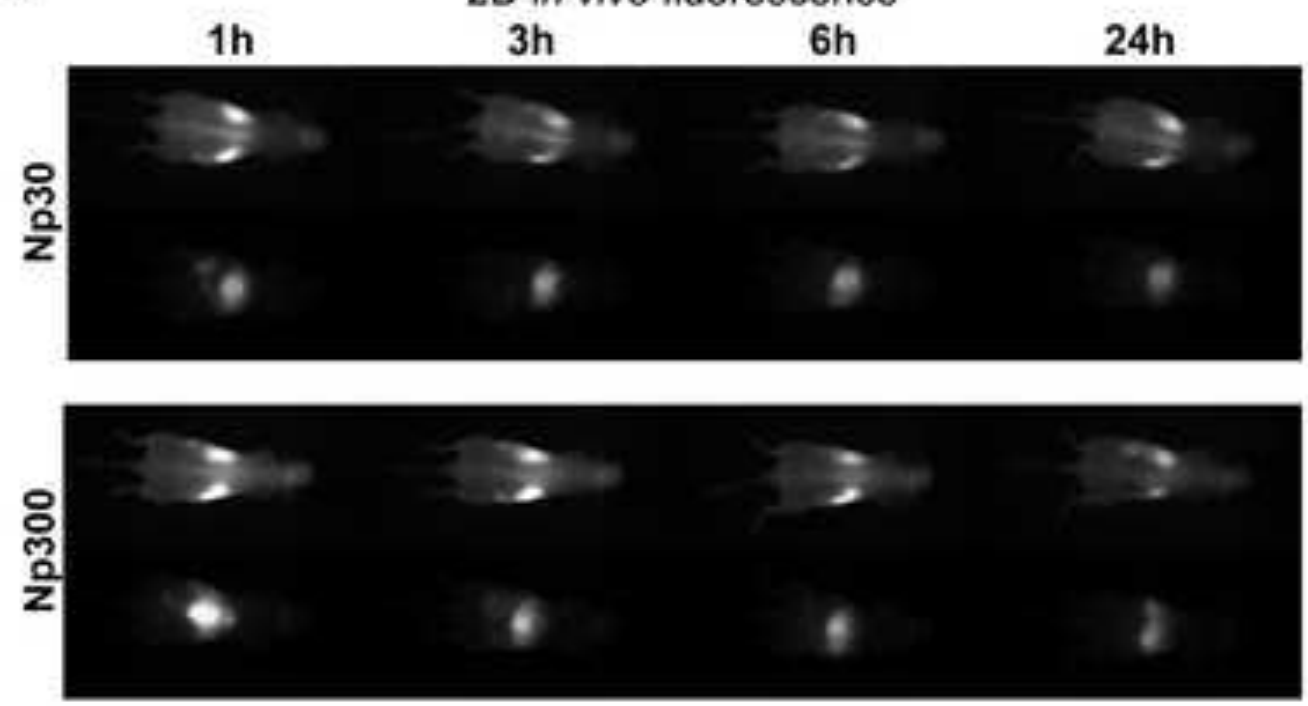

C

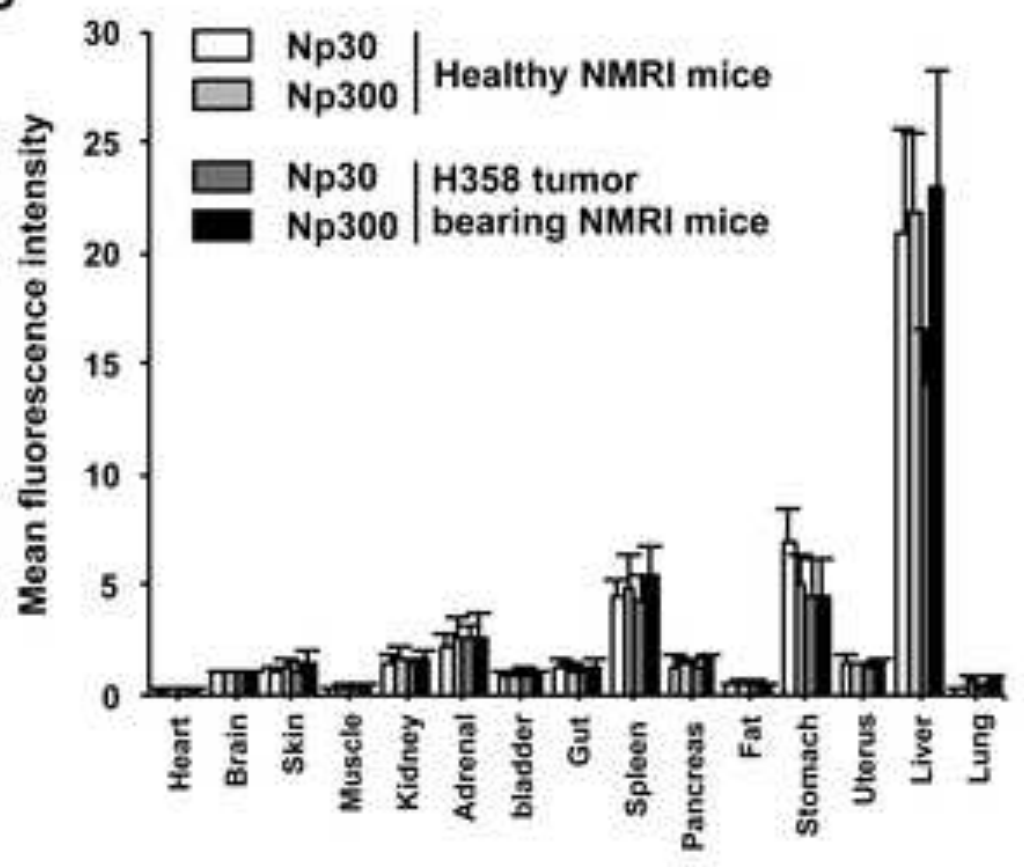

B 2D ex vivo fluorescence (24h)

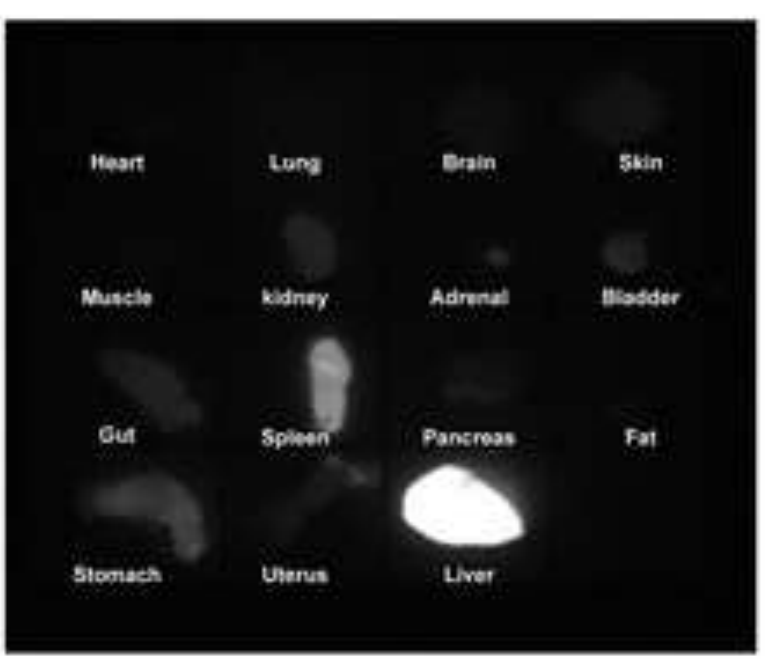

D

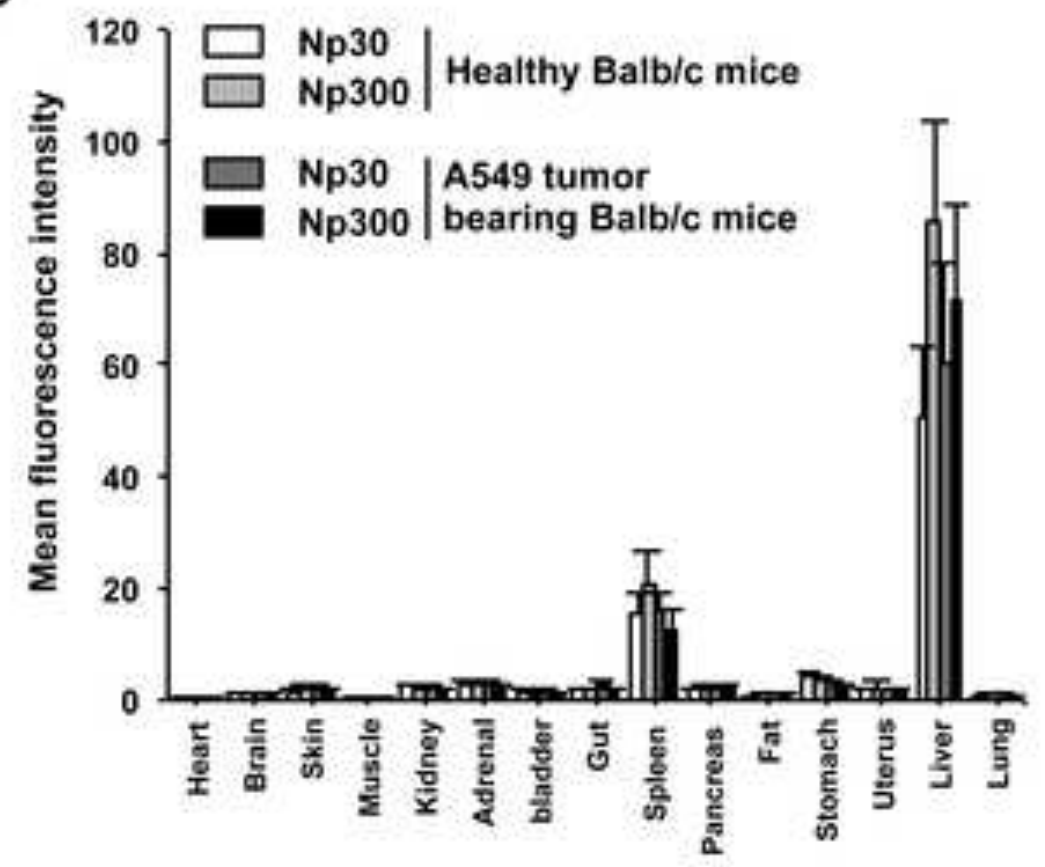




\section{Figure 4}

Click here to download high resolution image

\section{Figure 4}

A

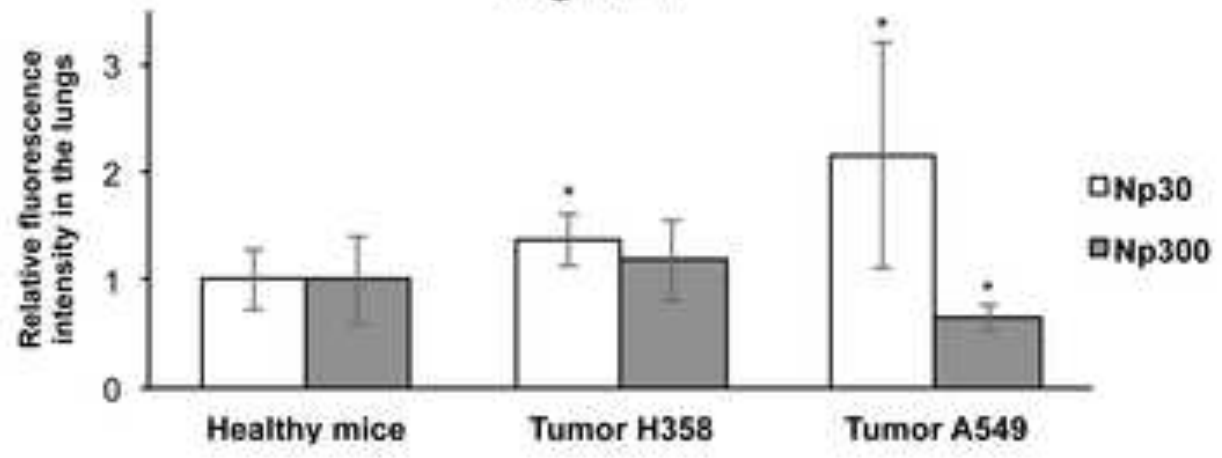

B

Ex vivo lung fluorescence and bioluminescence in $\mathrm{H} 358$ tumors
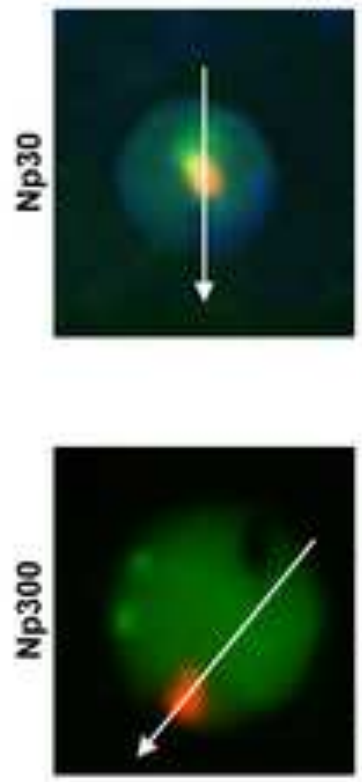

D
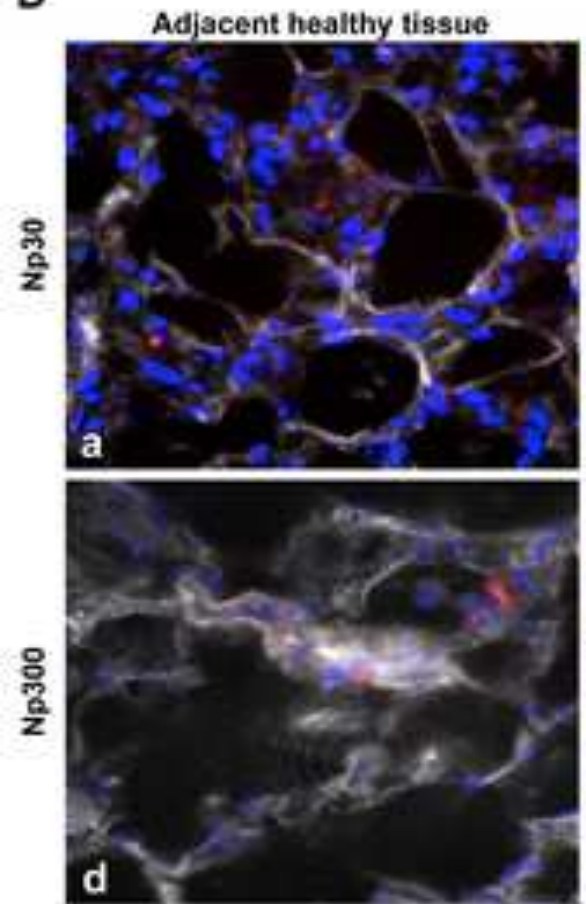

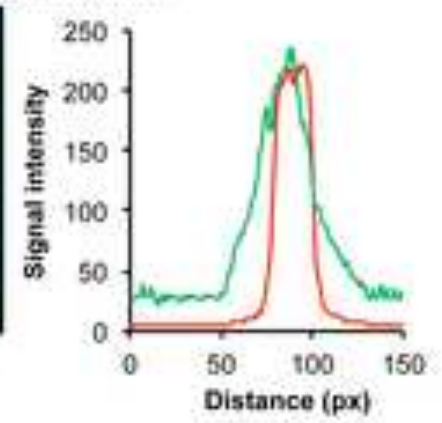

C Ex vivo lung fluorescence and bioluminescence in A549 tumors
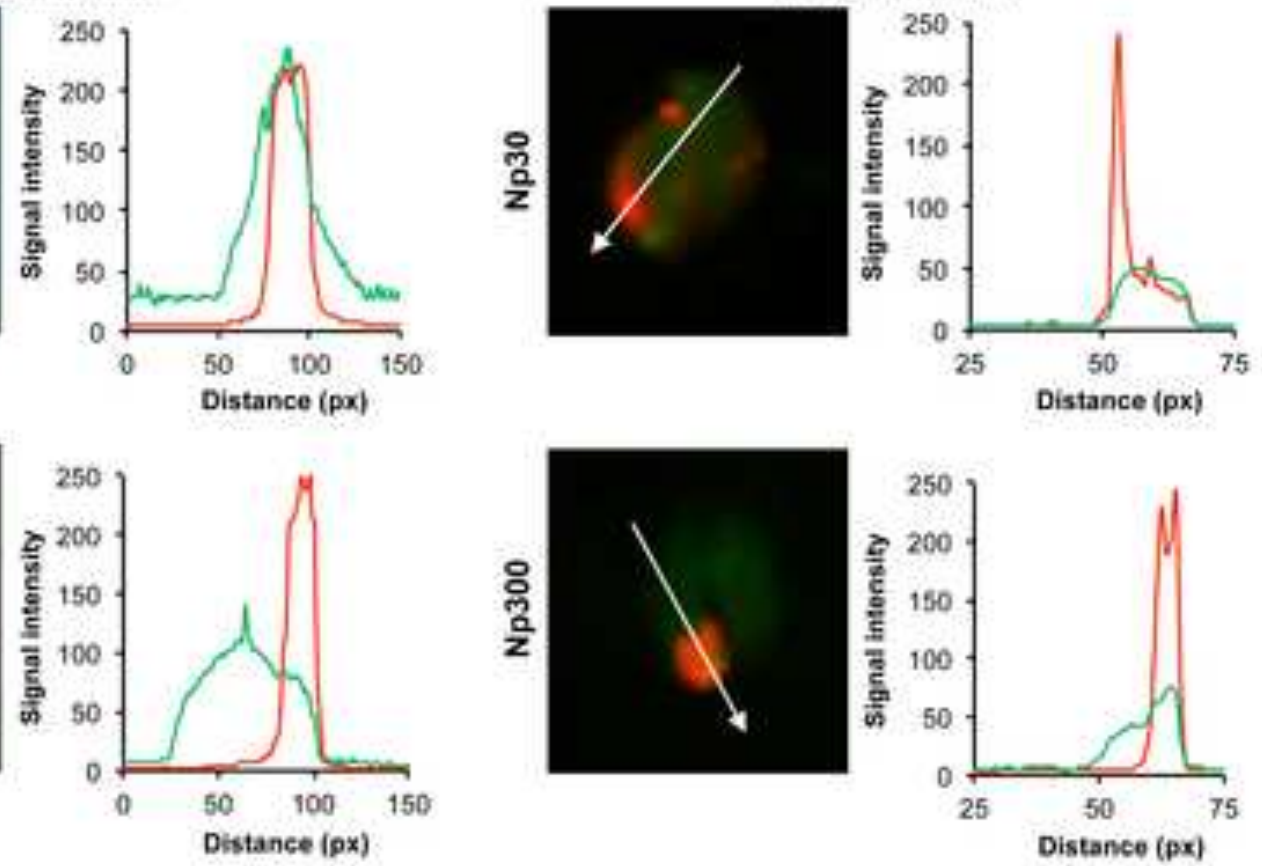
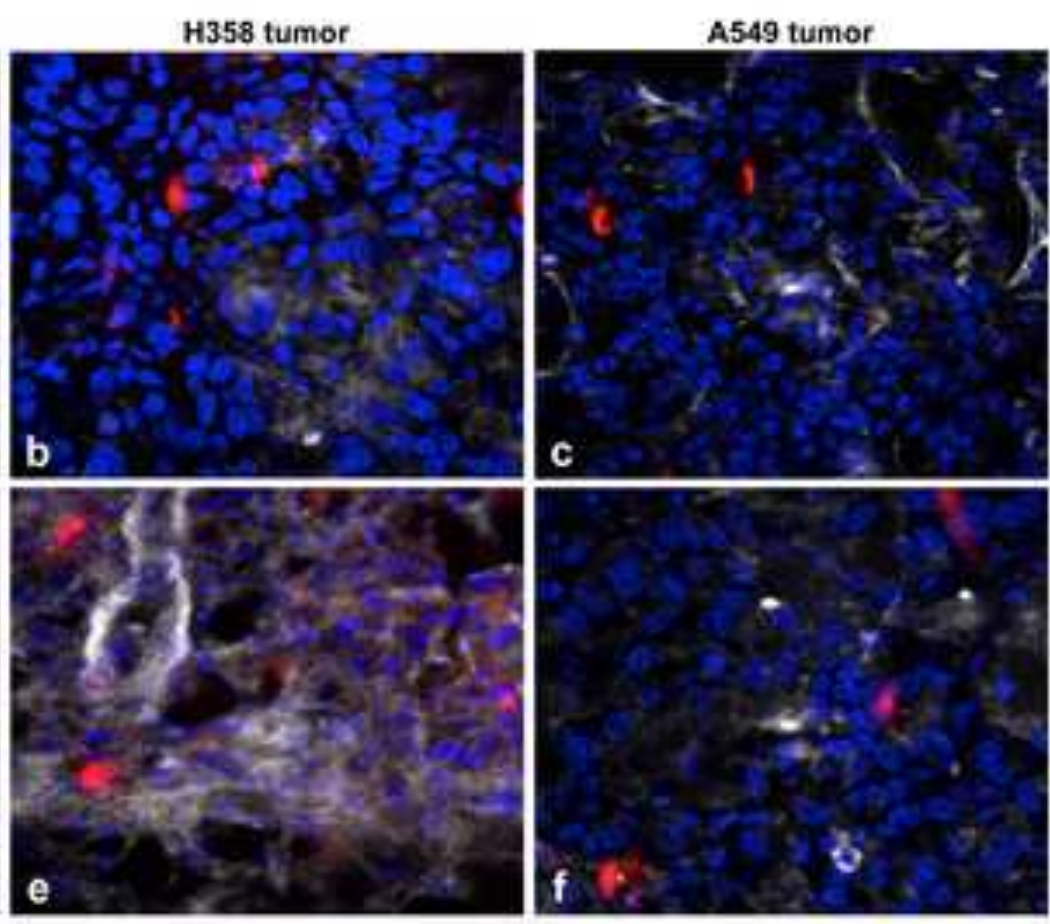
Figure 5

Intrapulmonary administration

A

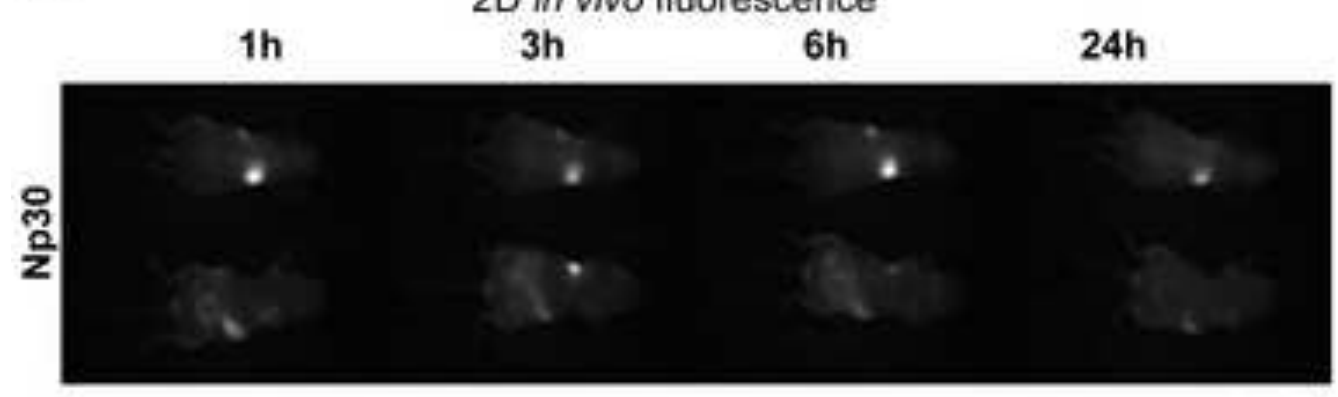

음

D
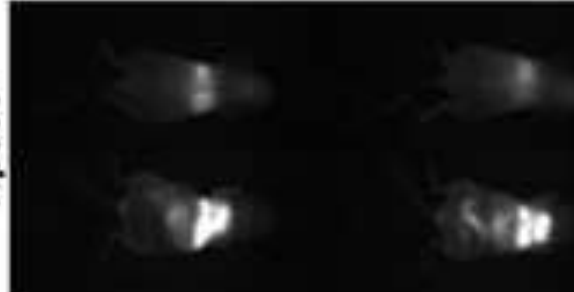

C

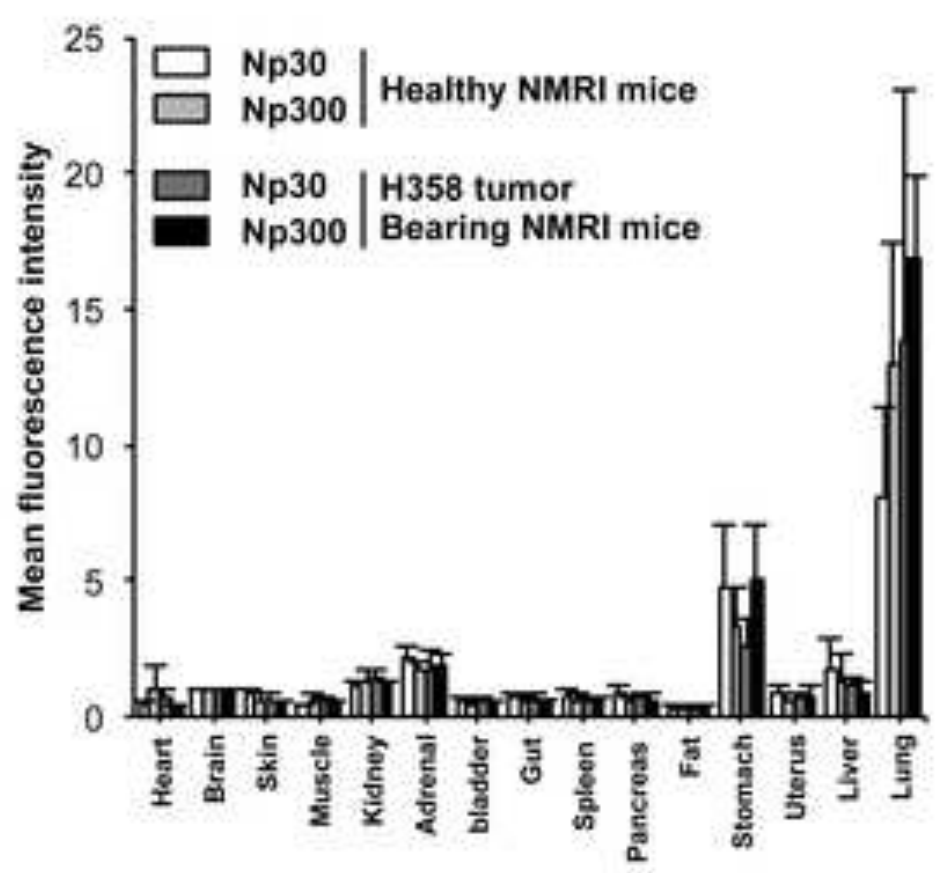

B

$2 \mathrm{D}$ ex vivo fluorescence (24h)

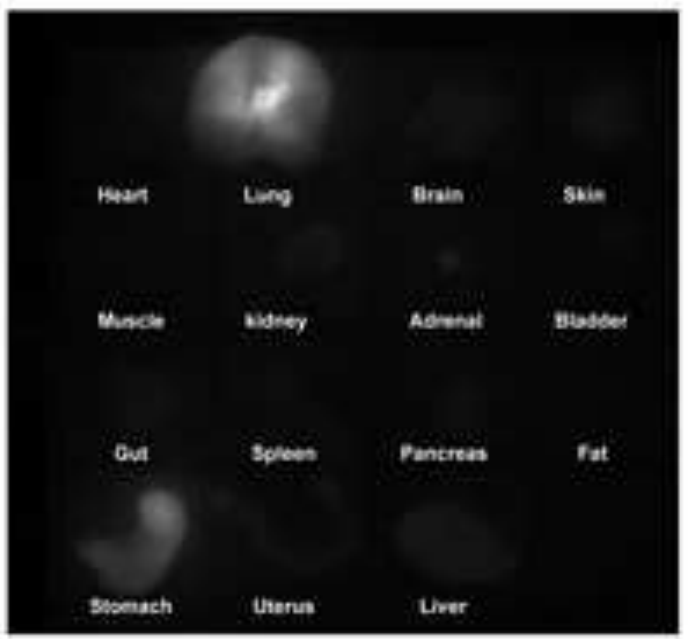

D

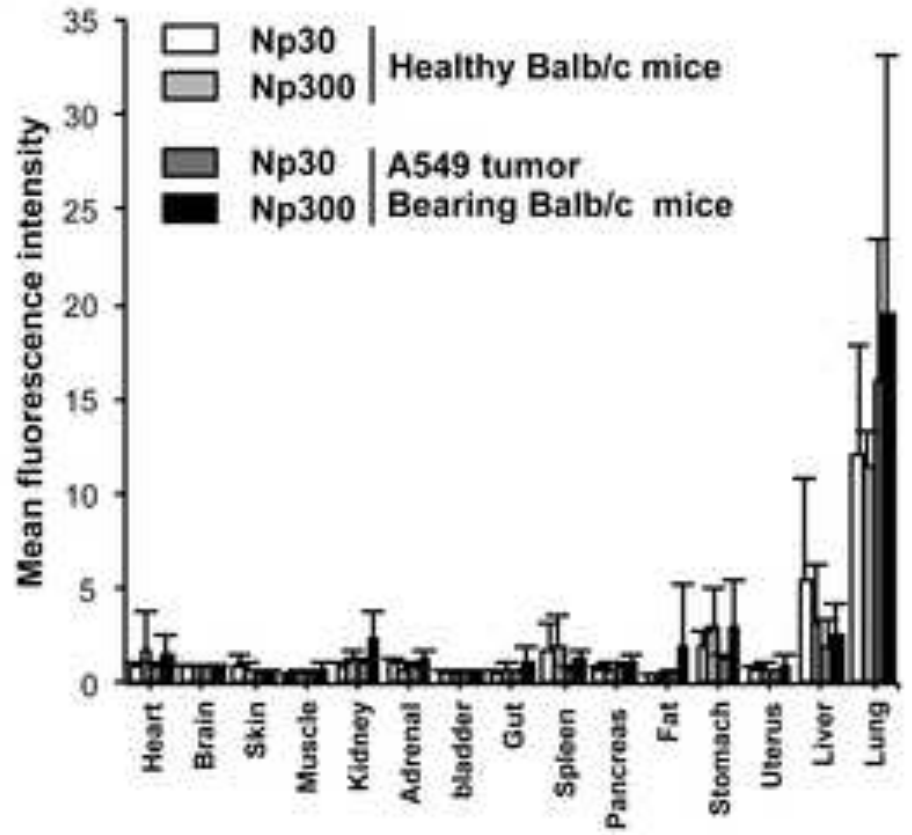




\section{Figure 6}

Click here to download high resolution image

\section{Figure 6}

A

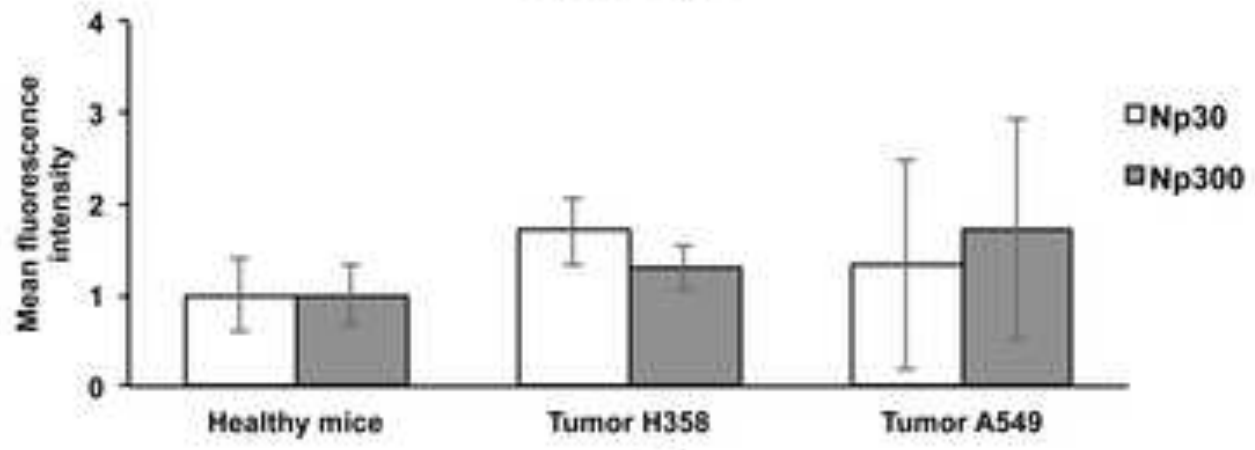

B

Ex vivo lung fluorescence and bioluminescence in $\mathrm{H} 358$ tumors
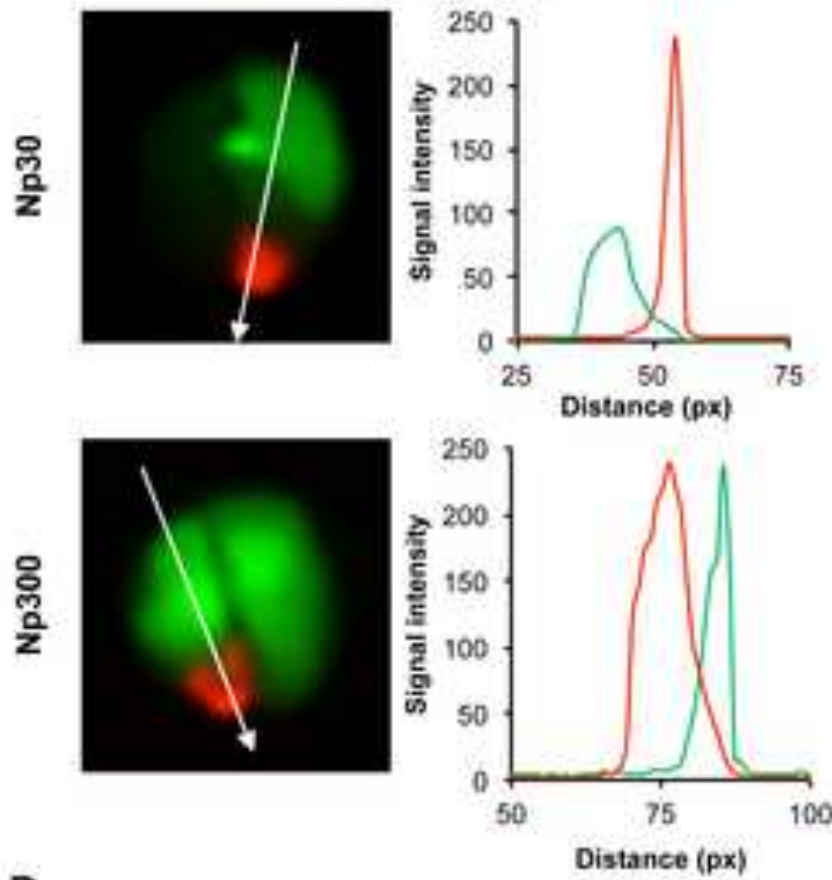

C

Ex vivo lung fluorescence and bioluminescence in A549 tumors
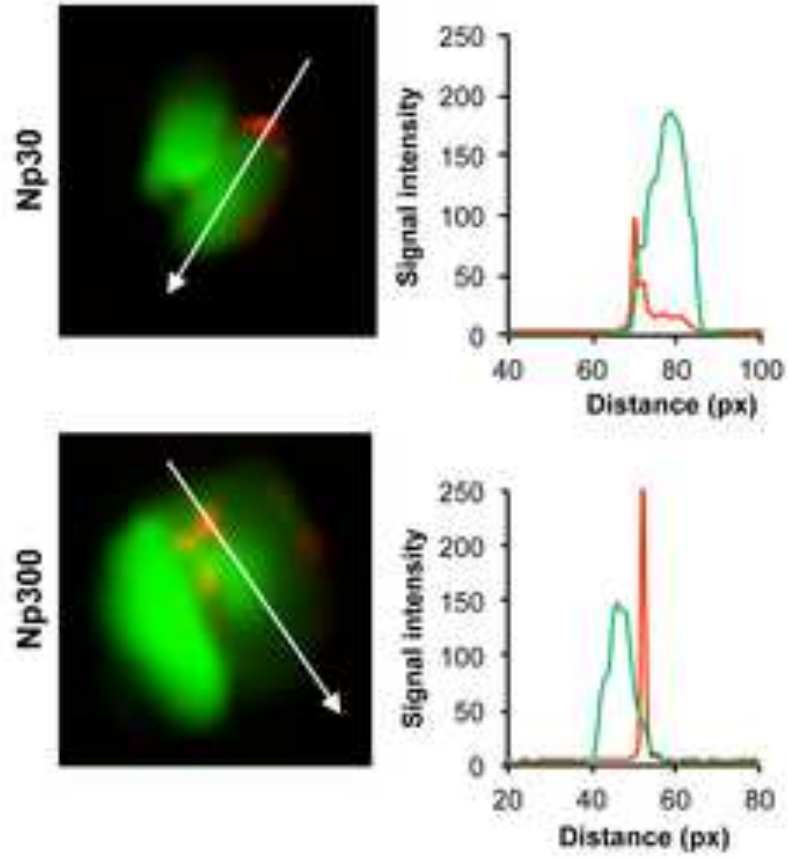

D
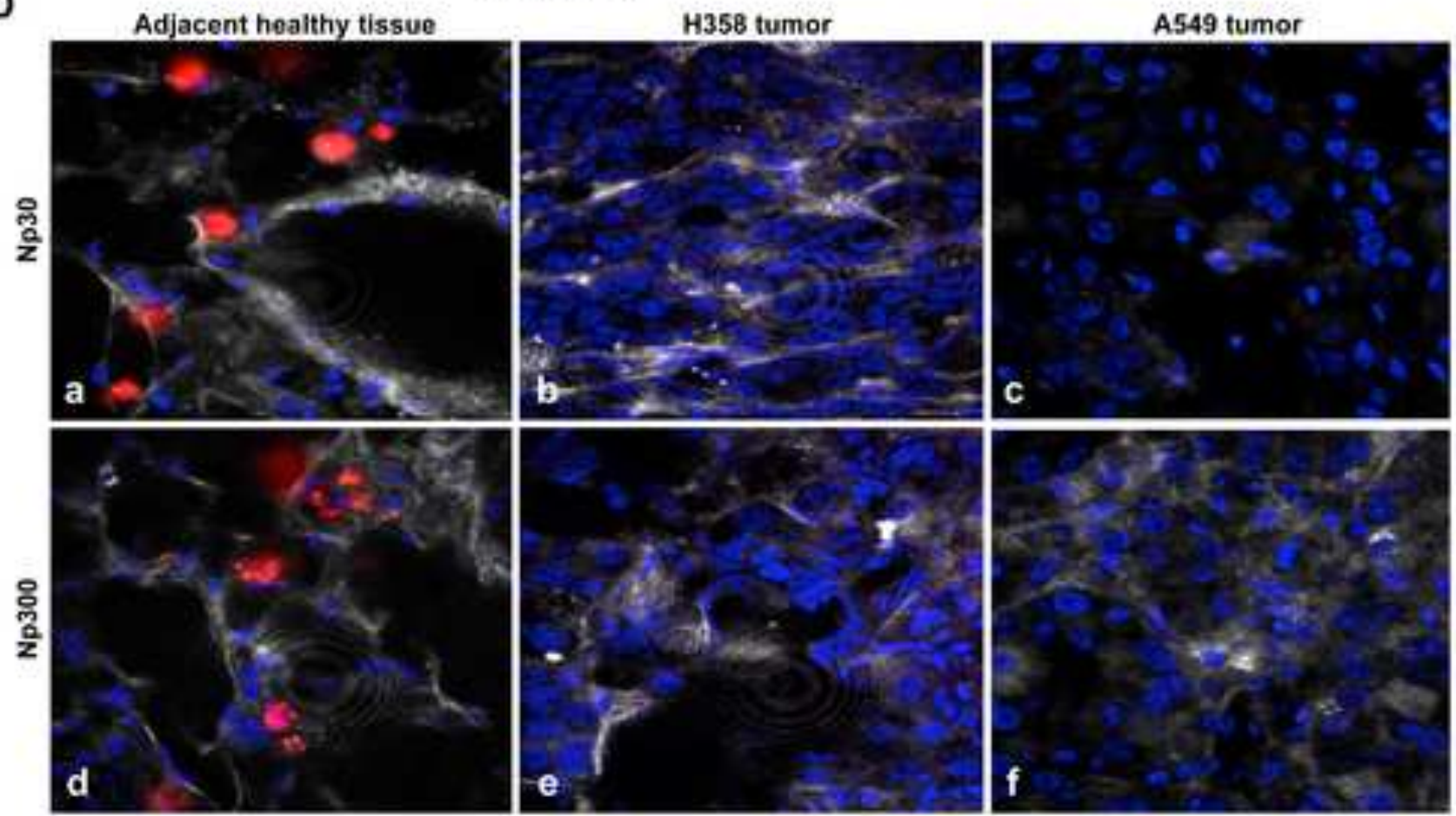
A

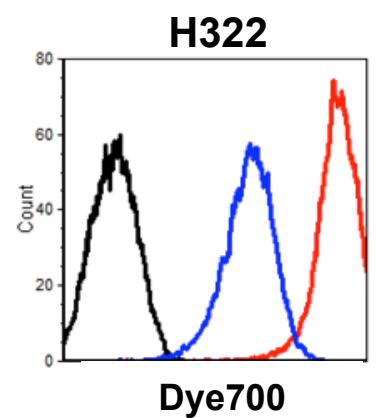

Dye700
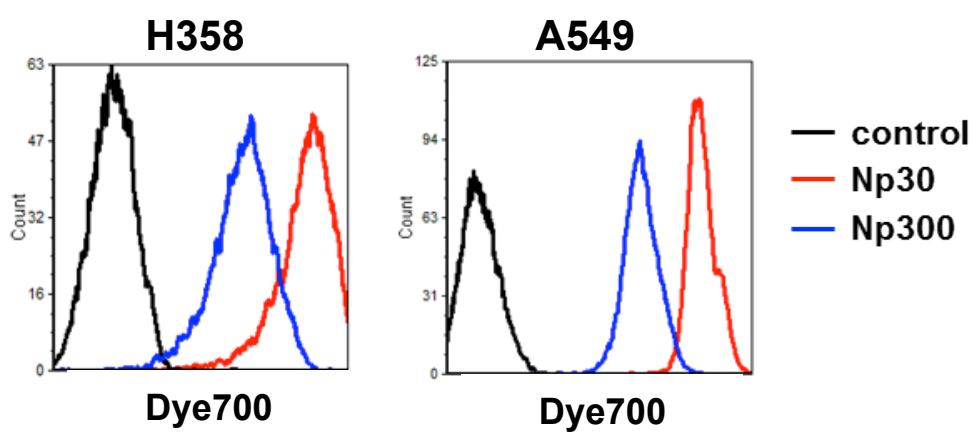

B

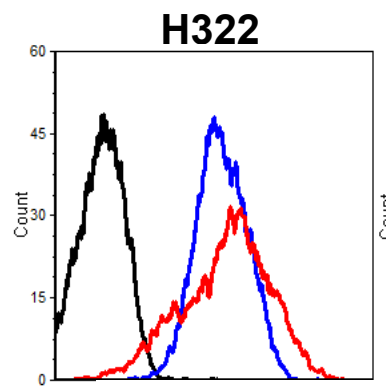

Dye700

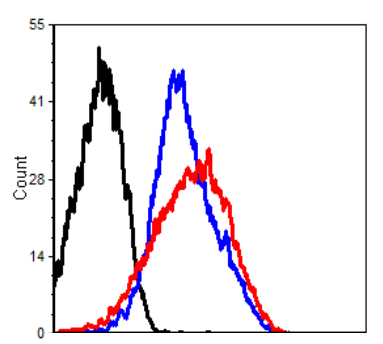

Dye700

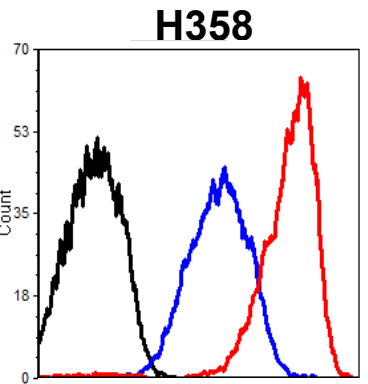

Dye700

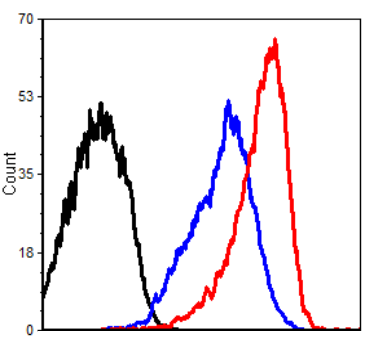

Dye700

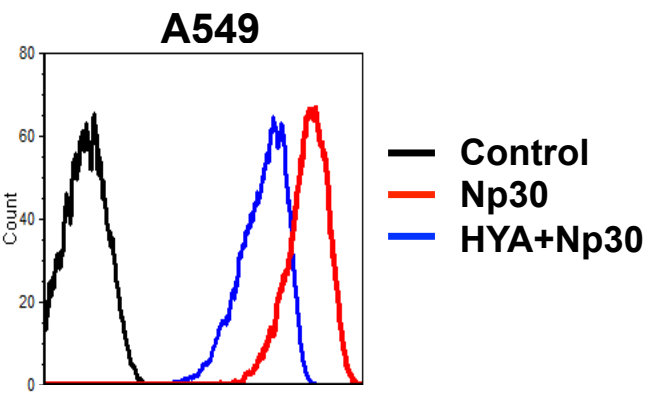

Dye700

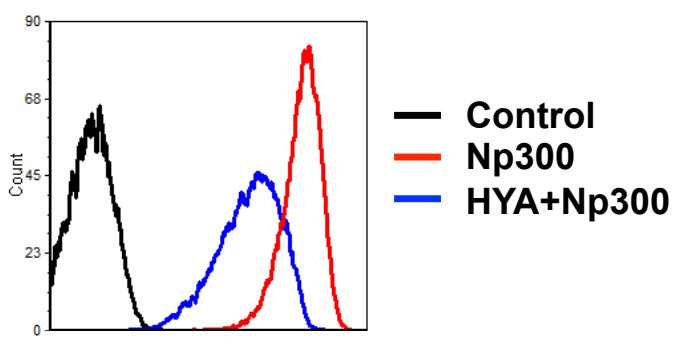

Dye700

\section{Supplementary Figure S1:}

A: Representative flow cytometry histograms showing H322, H358 and A549 cells stained with Dye700-labeled nanoparticles at $160 \mu \mathrm{g} / \mathrm{mL}$ (Np30, red curve; Np300, blue curve; no nanoparticle, black curve) after $30 \mathrm{~min}$ at $37^{\circ} \mathrm{C}$.

B: Representative flow cytometry histograms displaying the relative fluorescence intensity of unstained control H322, H358 or A549 cells (black curve), or cells treated with $8 \mu \mathrm{g} / \mathrm{mL} \mathrm{Np30} \mathrm{(upper}$ panel) or Np300 (lower panel) with (red curve) or without $5 \mathrm{mg} / \mathrm{mL}$ hyaluronan 175-300 kDa pretreatment (blue curve). 


\section{Supplementary Fig S2}

A

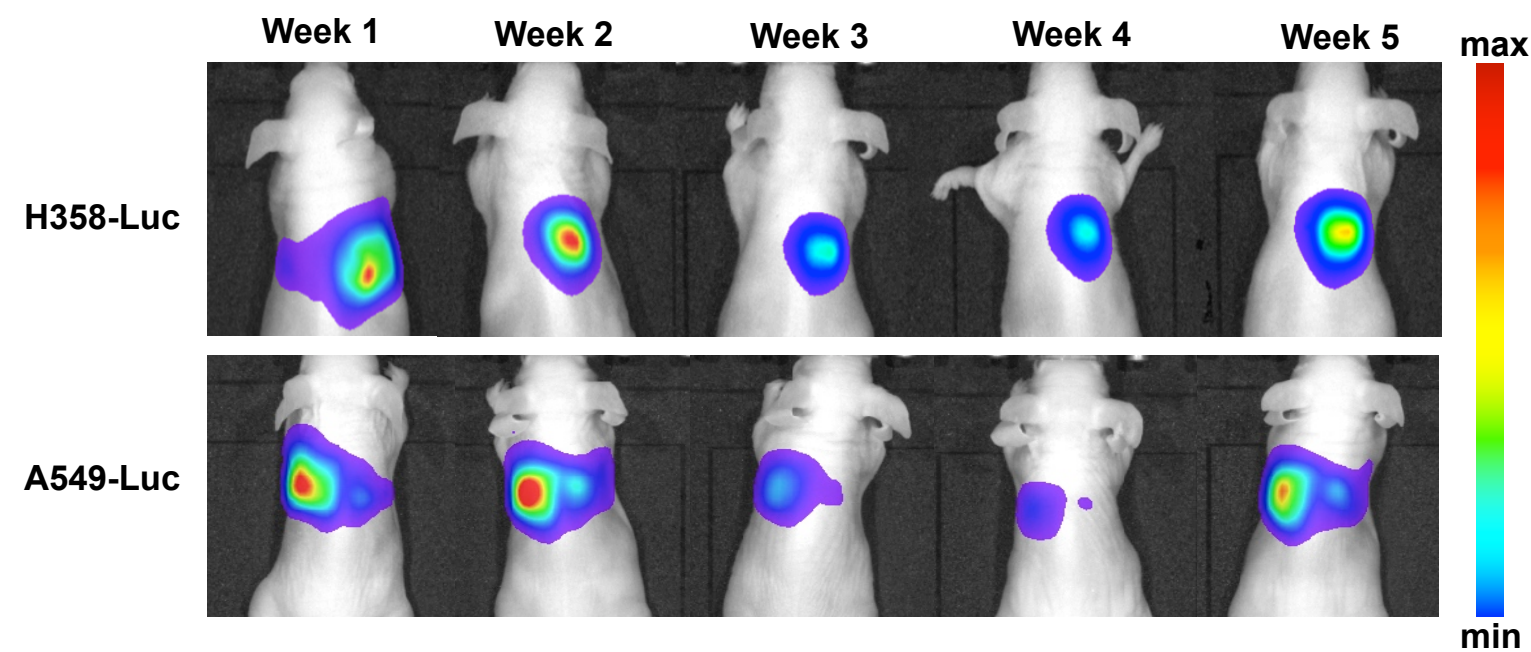

B $\quad$ H358

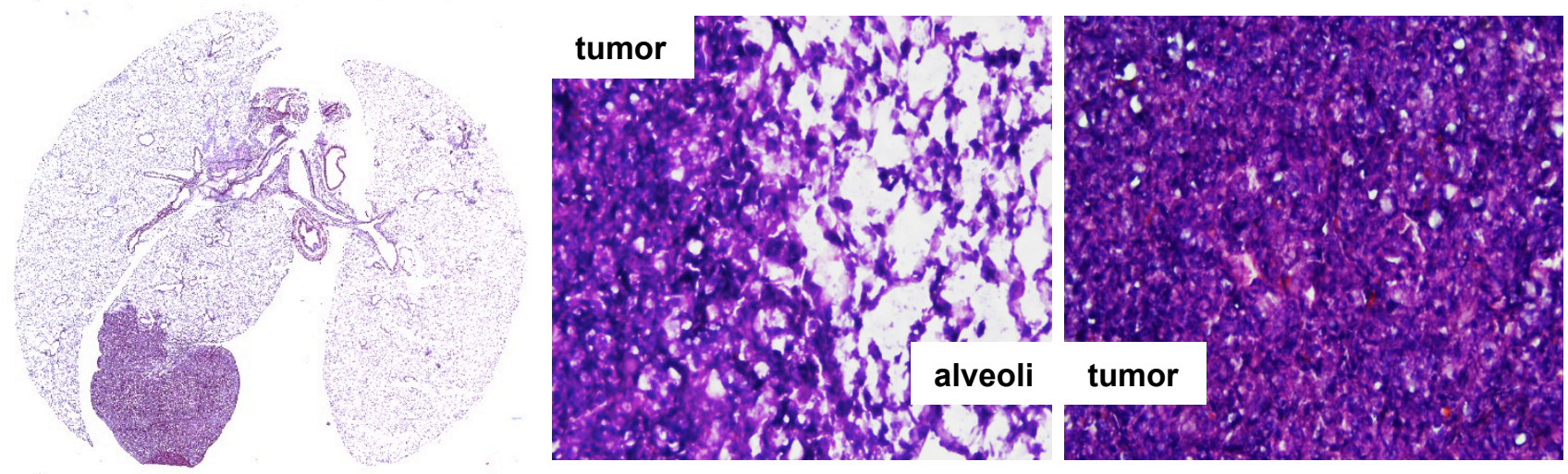

C $\quad$ A549
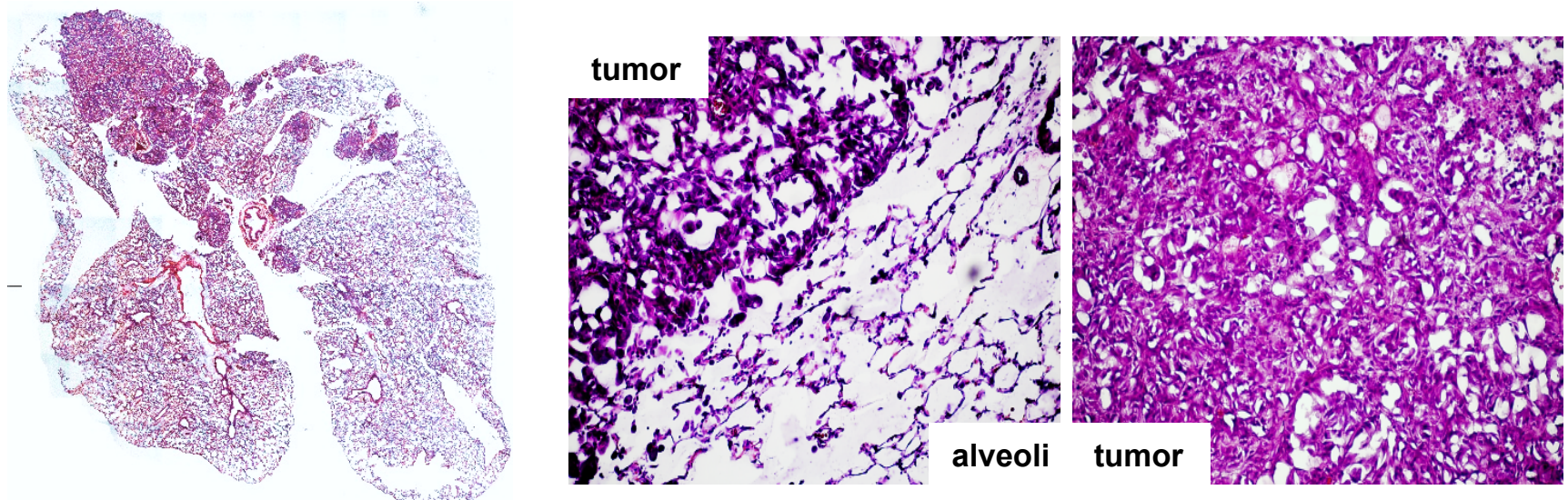

Supplementary Figure S2: H358 and A549 orthotopic lung cancer mouse models.

A: Lung tumors development of intrapulmonary inoculation of luciferase-modified human lung cancer H358 (H358-Luc) or A549 (A549-Luc) cells was followed using bioluminescence in vivo at the indicated times. B-C: Detection of lung tumor nodules on lung sections after hematoxylin and eosin staining in H358 (B) and A549 (C) orthotopic tumors. Images are at 4X (left) and 20X magnification (right). 


\section{Supplementary Fig S3}
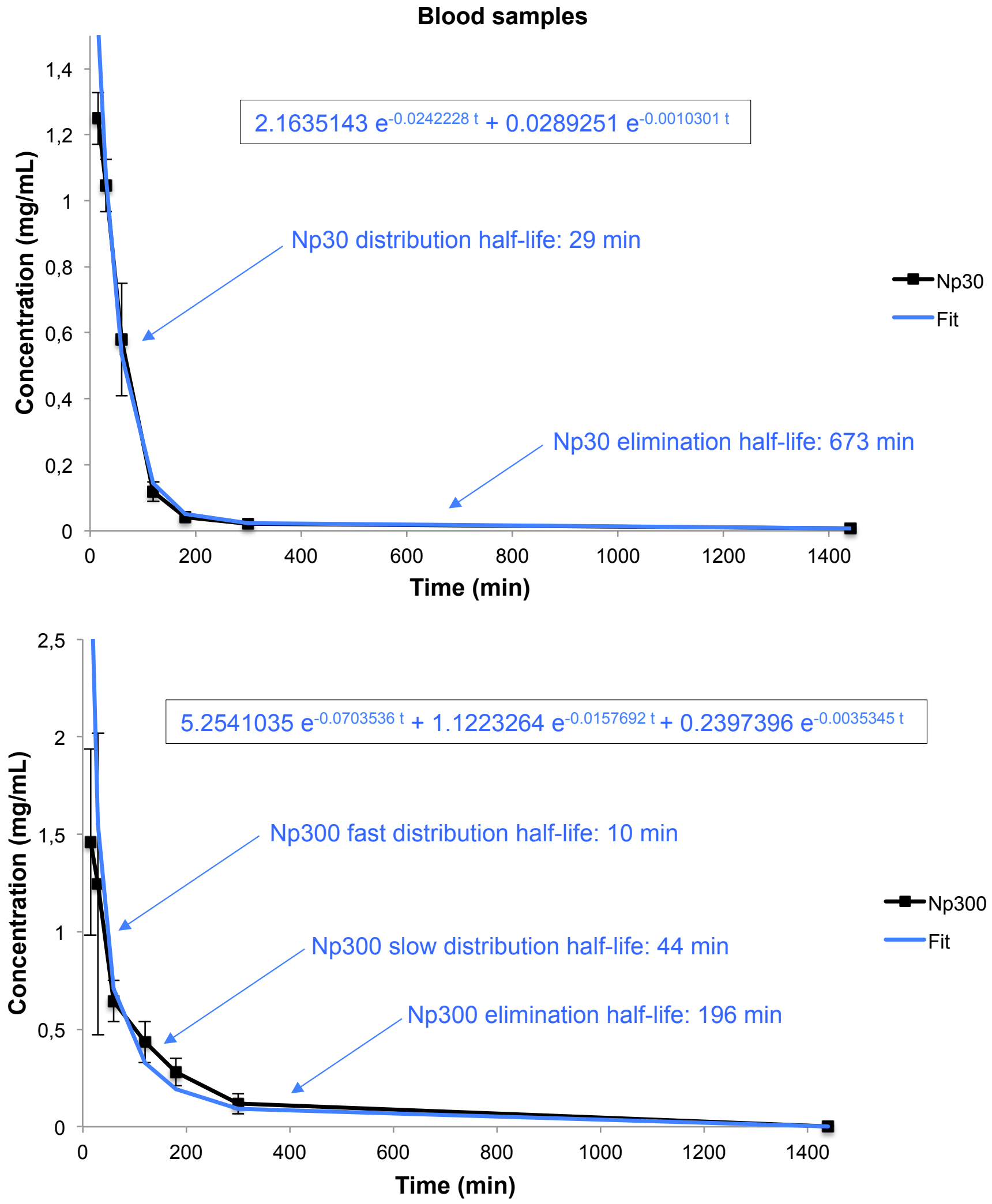

Supplementary Figure S3: Nanoparticles distribution and elimination blood imaging.

Healthy mice were injected intravenously with Dye700-labeled Np30 ( $=5)$ or Np300 (n=4). The fluorescence intensity measurements of blood samples were performed over time for each nanoparticle. The results of theses measurements are expressed as the mean \pm SD and the exponential decay parameters were fitted. 


\section{Supplementary Fig S4}
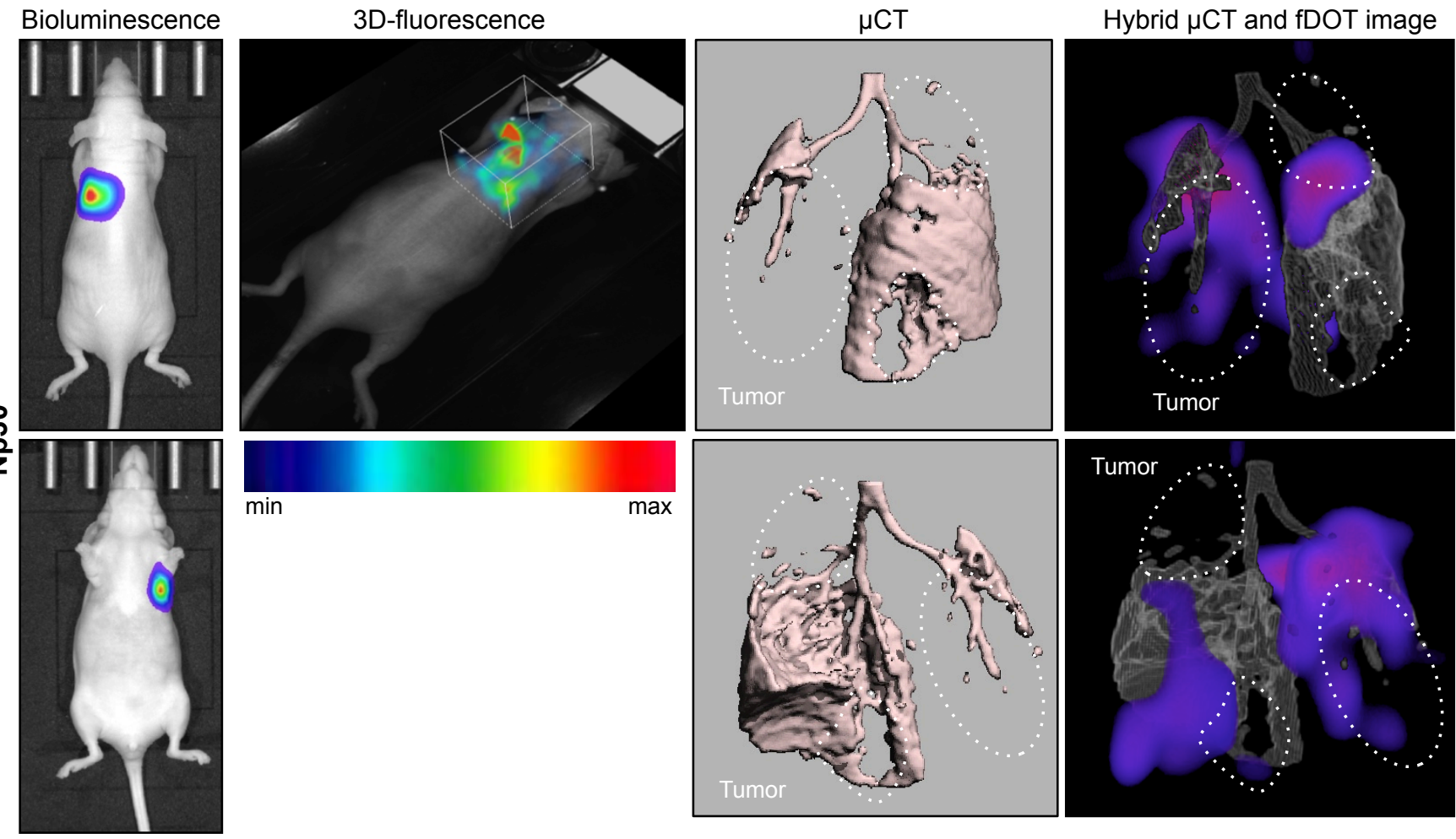

$\max$

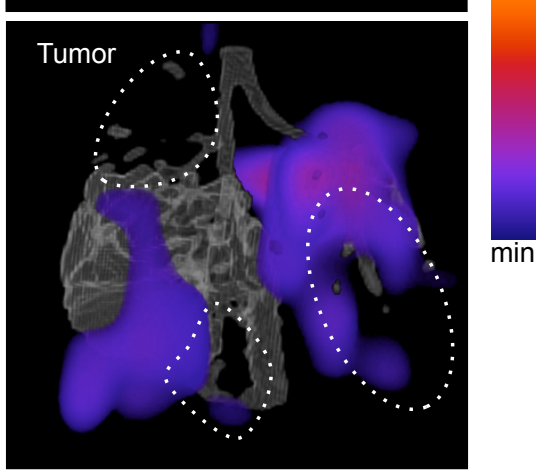

Bioluminescence

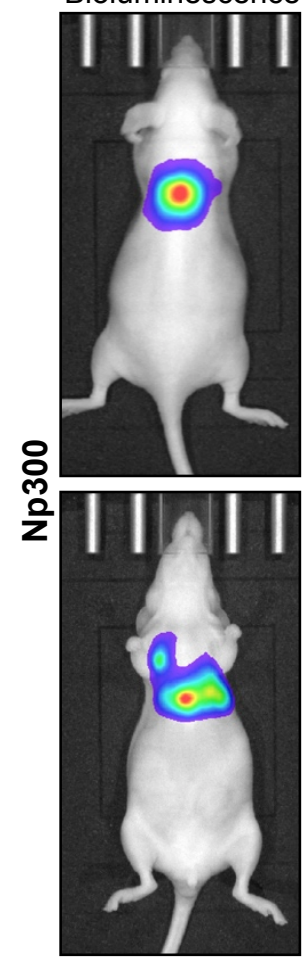

3D-fluorescence
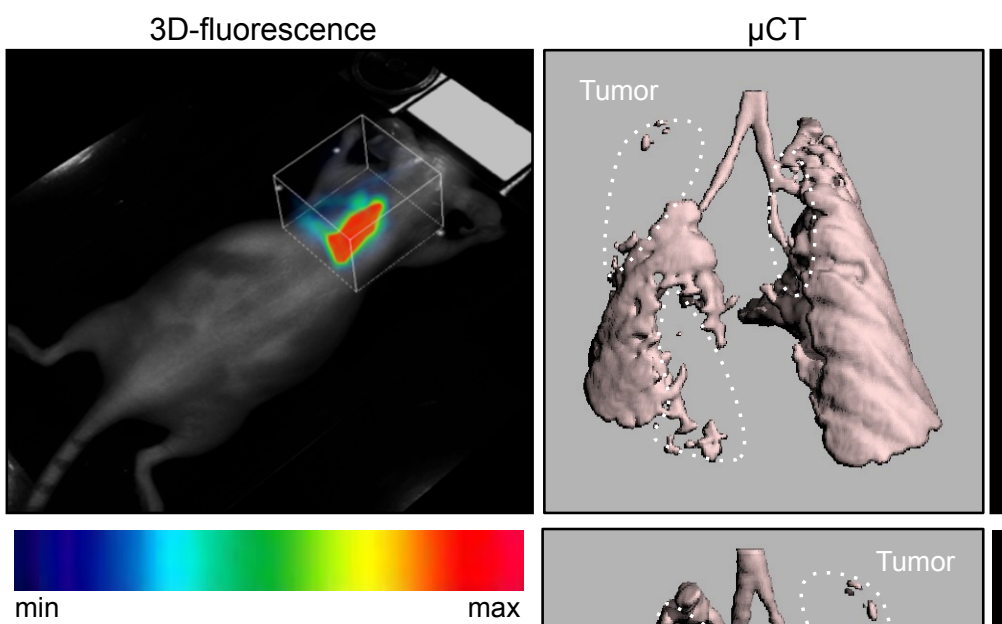

Hybrid $\mu$ CT and fDOT image
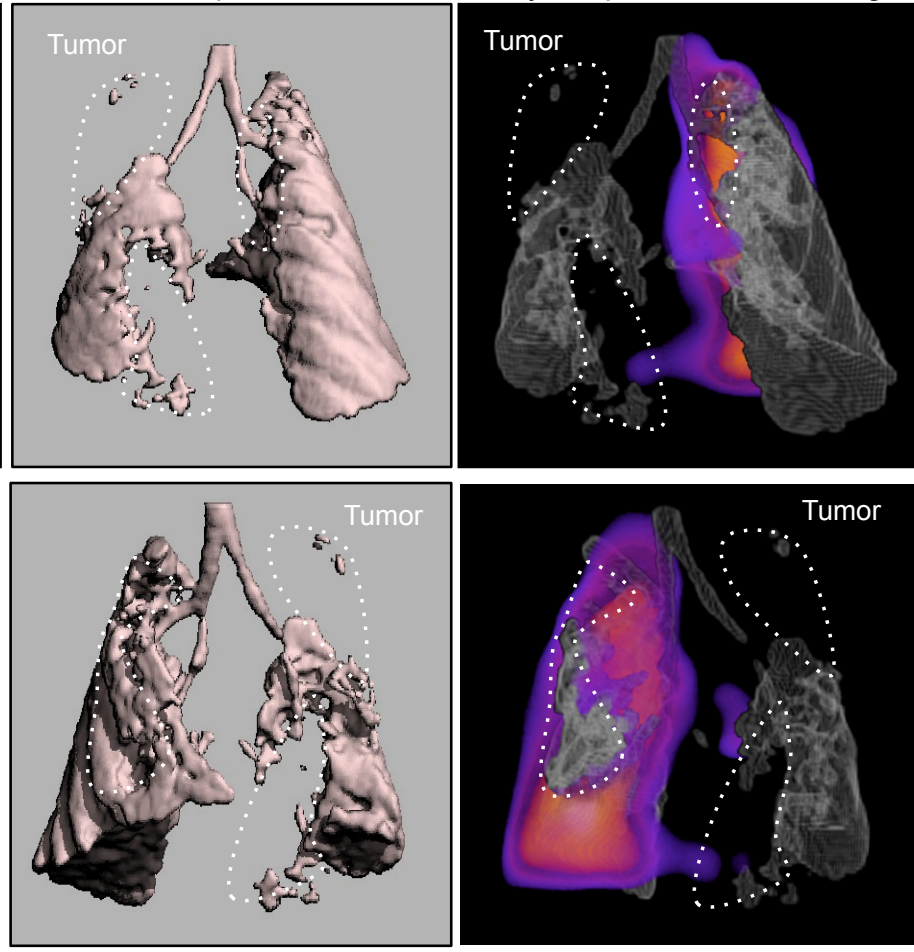

$\max$

\section{Supplementary Figure S4: In vivo H358-Luc orthotopic lung tumor imaging.}

Dy700 labeled Np30 or Np300 were administered intrapulmonary in H358 lung tumor-bearing mice. Bioluminescence, microCT, and 3D-fluorescence tomography imaging were performed $24 \mathrm{~h}$ after nebulization of nanoparticles. Bioluminescent signal showed H358-Luc tumor cells in vivo. 3D-fluorescent signal showed nanoparticles location in thoracic region. MicroCT analysis showing the air in the lungs, solid tumor location was observed through absence of signal on image of the lungs. The 3D fluorescent signal was merged with the mice's lungs in order to replace the fluorescent signal in an anatomical context. For each mouse, a dorsal view (upper line) and a ventral view (lower line) were shown. White dotted line: tumor site. 